\title{
Alpha rhythm induces attenuation-amplification dynamics in neural activity cascades
}

Fabrizio Lombardi ${ }^{\mathrm{a}}$, Hans J. Herrmann ${ }^{\mathrm{b}}$, Liborio Parrino ${ }^{\mathrm{c}}$, Dietmar Plenz ${ }^{\mathrm{d}}$, Silvia Scarpetta $^{\mathrm{e}, \mathrm{f}}$, Anna Elisabetta Vaudano ${ }^{\mathrm{g}, \mathrm{h}}$, Lucilla de Arcangelis ${ }^{\mathrm{i}}$, and Oren Shriki ${ }^{\mathrm{l}}$

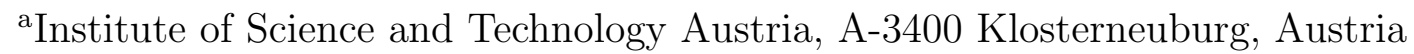

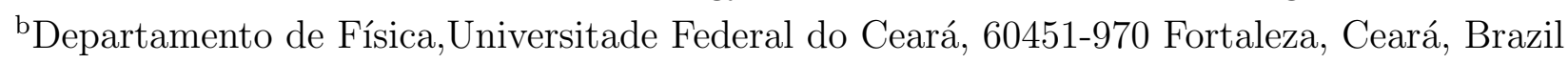

'Sleep Disorders Center, Department of Neurosciences, University of Parma, Italy

${ }^{\mathrm{d}}$ Section on Critical Brain Dynamics, NIH, Bethesda, Maryland 20892, USA

eDepartment of Physics, University of Salerno, Italy

${ }^{\mathrm{f}}$ INFN sez. Napoli Gr. Coll. Salerno, Italy

gNeurology Unit, Azienda Ospedaliero-Universitaria of Modena, OCB Hospital, Modena, Italy

${ }^{\mathrm{h}}$ Department of Biomedical, Metabolic and Neural Sciences, University of Modena and

Reggio Emilia, Modena, Italy

${ }^{i}$ Department of Engineering, University of Campania "Luigi Vanvitelli", 81031 Aversa(CE), Italy

${ }^{1}$ Department of Brain and Cognitive Sciences, Ben-Gurion University of the Negev, Beer-Sheva, Israel

\begin{abstract}
Rhythmical cortical activity has long been recognized as a pillar in the architecture of brain functions. Yet, the dynamic organization of its underlying neuronal population activity remains elusive. Here we uncover a unique organizational principle regulating collective neural dynamics associated with the alpha rhythm in the awake resting-state. We demonstrate that cascades of neural activity obey attenuation-amplification dynamics (AAD), with a transition from the attenuation regime - within alpha cycles - to the amplification regime - across a few alpha cycles - that correlates with the characteristic frequency of the alpha rhythm. We find that this short-term AAD is part of a large-scale, size-dependent temporal structure of neural cascades that obeys the Omori law: Following large cascades, smaller cascades occur at a rate that decays as a power-law of the time elapsed from such events - a long-term AAD regulating brain activity over the timescale of seconds. We show that such an organization corresponds to the "waxing and waning" of the alpha rhythm. Importantly, we observe that short- and long-term AAD are unique to the awake resting-state, being absent during NREM sleep. These results provide a quantitative, dynamical description of the so-far-qualitative notion of the "waxing and waning" phenomenon, and suggest the AAD as a key principle governing resting-state dynamics across timescales.
\end{abstract}

\section{Introduction}

The mammalian brain exhibits complex rhythmic dynamics that span a broad range of frequencies [8]. Brain rhythms emerge as periodic amplitude fluctuations in electro-physiological recordings, which 
result from the synchronous activation of large populations of neurons [7, 9]. Oscillations in distinct frequency bands have been associated with different brain functions and physiologic states [7, 50, 3]. Among brain rhythms, oscillations in the alpha band (8-13 Hz) play a prominent role in human brain activity. Characteristic of the eyes closed awake resting-state, alpha oscillations have been associated with the processes of task disengagement [46, particularly in visual processing, as well as functional inhibition, suppression of visual activity through pulsed inhibition [37], facilitation of periodic sampling of visual information, and, more generally, with propagation of activity throughout the brain and feedback processing within and across brain regions [54, 56, 55]. Indeed, a number of studies indicate that changes in information processing and cognitive performance are accompanied by rhythmic modulation of alpha amplitude and power, pointing in particular to an inverse relationship between alpha activity and neural firing - an observation central to the hypothesis of pulsed inhibition [46, 37]. Amplitude fluctuations occur across multiple timescales, a distinctive feature of alpha oscillations known as "waxing and waning" [41, 40]. They show non-trivial features, such as long-range temporal correlations (LRTC) [27, 43] and non-Gaussian bimodal distributions of power [19], suggesting the existence of two distinct modes - higher- and lower-power modes [19].

Several models have been proposed to explain the emergence of alpha oscillations and their dynamic characteristics, from mutually coupled excitatory (E) and inhibitory (I) spiking and stochastic neurons [68, 48, to more realistic thalamic and corticothalamic mechanistic models [65, 64, 20, 15]. In particular, a recently proposed probabilistic integrate-and-fire (IF) spiking model with E and I neurons generates long-range correlated fluctuations reminiscent of alpha oscillations in the resting state and consistent with the notion of critical brain dynamics [48]. Taking into account corticothalamic loop mechanisms, the neural field model by Freyer et al. [20] reproduces spontaneous jumps between lower- and higher-power alpha modes in the presence of a subcritical Hopf bifurcation.

However, neural dynamics and generative mechanisms underlying emergent alpha rhythms remain, to a large extent, not known and highly debated. Empirical evidence indicates that neural oscillations coexist with cascades of neural activity termed neuronal avalanches, which are spatio-temporal patterns of activity with no characteristic size, time, or spatial scale [4, 22, 32, 60, 28, 43, 39]. The observations of neuronal avalanches across species and systems with similar features in populations of spiking neurons, as well as in coarse-grained measures of neural activity [4, 45, 151, 60, 35, 38, 44, 49, 70, 18], suggest they represent a fundamental organizational principle of brain network dynamics.

Yet, the relationship between emergent brain rhythms and the cascading process defining neuronal avalanches is currently scarcely understood. On the one hand, brain rhythms have characteristic times and amplitudes, and are, by definition, a property of the integrated electromagnetic signals arising from the superposition of synaptic currents from large neural populations. On the other hand, neuronal avalanches exhibit scale-free, power-law statistical properties [4], and show a consistent spatio-temporal organization in terms of discrete events, from sequences of spiking neurons [44, 38, 49, 70, 18, to clusters of extreme amplitude fluctuations in LFP, EEG, and MEG sensor arrays [4, 45, 60, 35]. Such a coexistence of scale-free avalanches and scale-specific oscillations has been the focus of recent modelling attempts [48, 59, 58, 34].

By dissecting brain activity across resting wake and sleep, here, we uncover the relationship between dynamics of neural activity cascades and key features of brain oscillations. In particular, we demonstrate that the alpha rhythm is associated with peculiar attenuation-amplification dynamics (AAD) in resting-state brain activity - a signature of pulsed inhibition governing collective neural activity across multiple timescales. The AAD of the resting state is encompassed by a precise structure 
of the cascading dynamics that is dictated by large cascades, which correspond to high amplitude alpha bursts, and shows the characteristic time of the alpha "waxing and waning" [41, 66, 19]. This size-dependent temporal structure is described by the Omori law [63]. Crucially, we show that brain activity during sleep does not exhibit AAD and does not obey the Omori law, but follows stretched exponential dynamics pointing to a radically different, multi-site-governed underlying cascading process.

\section{Results}

\subsection{Dynamics of neural activity cascades during the awake resting-state}

To characterize collective brain dynamics in relation to dominant brain rhythms, we analyzed MEG and EEG recordings of the spontaneous brain activity (Materials and Methods). First, we proceeded to identify spatio-temporal cascades of neural activity across the sensor arrays. To this end, we mapped the continuous signals into sequences of discrete events defined as the extremes of large positive and negative signal deflections exceeding an amplitude threshold $h$ (Materials and Methods) (Fig. 1A). This procedure preserves most of the collective features encoded in the continuous signals across the sensor array [61, 60, 35].

In Fig. 1A, we show a representative raster of events. The raster plot indicates that events tend to cluster in time across sensors - or cortical locations. We define a neural cascade, or avalanche, $a_{i}$ as a sequence of consecutive time bins with at least one event, preceded and followed by at least one empty bin (Fig. 1A) [4]. To each avalanche, we associate a size, $s_{i}$, which is given by the number of events occurring in the time bins that belong to it.

It has been recently shown that the distribution $P(s)$ of avalanche sizes follows a power-law behavior - $P(s) \propto s^{-\tau}$-both in MEG and EEG recordings [60, 35, 43]. Power-law distributions indicate an absence of characteristic scales, a property in stark contrast with the main essence of brain rhythms, namely, characteristic times and amplitudes [7, 8, 9].

The scale-free avalanche size distribution does not suggest any potential link with neural oscillations. To characterize the dynamics of avalanches and detect connections with coexisting rhythmic patterns, we turn our attention to the distribution of quiet times $\Delta t$ that separate consecutive avalanches (Figs. 1B,C). This distribution provides key information on the dynamics underlying emergent collective behaviors, which is not otherwise accessible, and is widely used across fields and disciplines [10, 63, 23, 13, 1, 31, 32, 29, 67]. The quiet time $\Delta t$ is defined as the time interval between the ending time $t_{i}^{e}$ of the avalanche $a_{i}$ and the starting time $t_{i+1}^{s}$ of the following avalanche $a_{i+1}$, namely, $\Delta t=t_{i+1}^{s}-t_{i}^{e}$ (Fig. 11). We find that for $\Delta t<1 \mathrm{~s}$, the distributions $P(\Delta t)$ are well described by two distinct power-law regimes: regime (A) for $\Delta t<100 \mathrm{~ms}$, with exponent $\mu_{A} \simeq 0.8$; and regime (B) for $100 \mathrm{~ms}<\Delta t<1000 \mathrm{~ms}$, with exponent $\mu_{B} \simeq 2.1$ (Figs. 1B,C). Importantly, the transition from power-law regime (A) to power-law regime (B) occurs around $\Delta t \simeq 100 \mathrm{~ms}$, which corresponds to the characteristic time of the alpha rhythm. We observe such behavior both in short MEG (4 min) (Fig. 1B) and EEG (3 min) recordings (Fig. 1 $\mathrm{C}$ ), as well as in prolonged (40 min) MEG recordings of the awake resting-state (Fig. 1B). Power-law regimes (A) and (B) are followed by a faster decay of the probability $P(\Delta t)$ for $\Delta t$ 's longer than $1 \mathrm{~s}$, which may be related to delta oscillations. The non-exponential, power-law quiet time distribution implies that neural cascades of activity are strongly correlated [63, 12, 35, 31, 33, 30]. Indeed, the distribution $P(\Delta t)$ calculated after random phase shuffling (Materials and Methods) of the original brain signals is exponential (Figs. 1 B,C). 
bioRxiv preprint doi: https://doi.org/10.1101/2022.03.03.482657; this version posted March 4, 2022. The copyright holder for this preprint (which was not certified by peer review) is the author/funder, who has granted bioRxiv a license to display the preprint in perpetuity. It is made available under aCC-BY-NC-ND 4.0 International license.
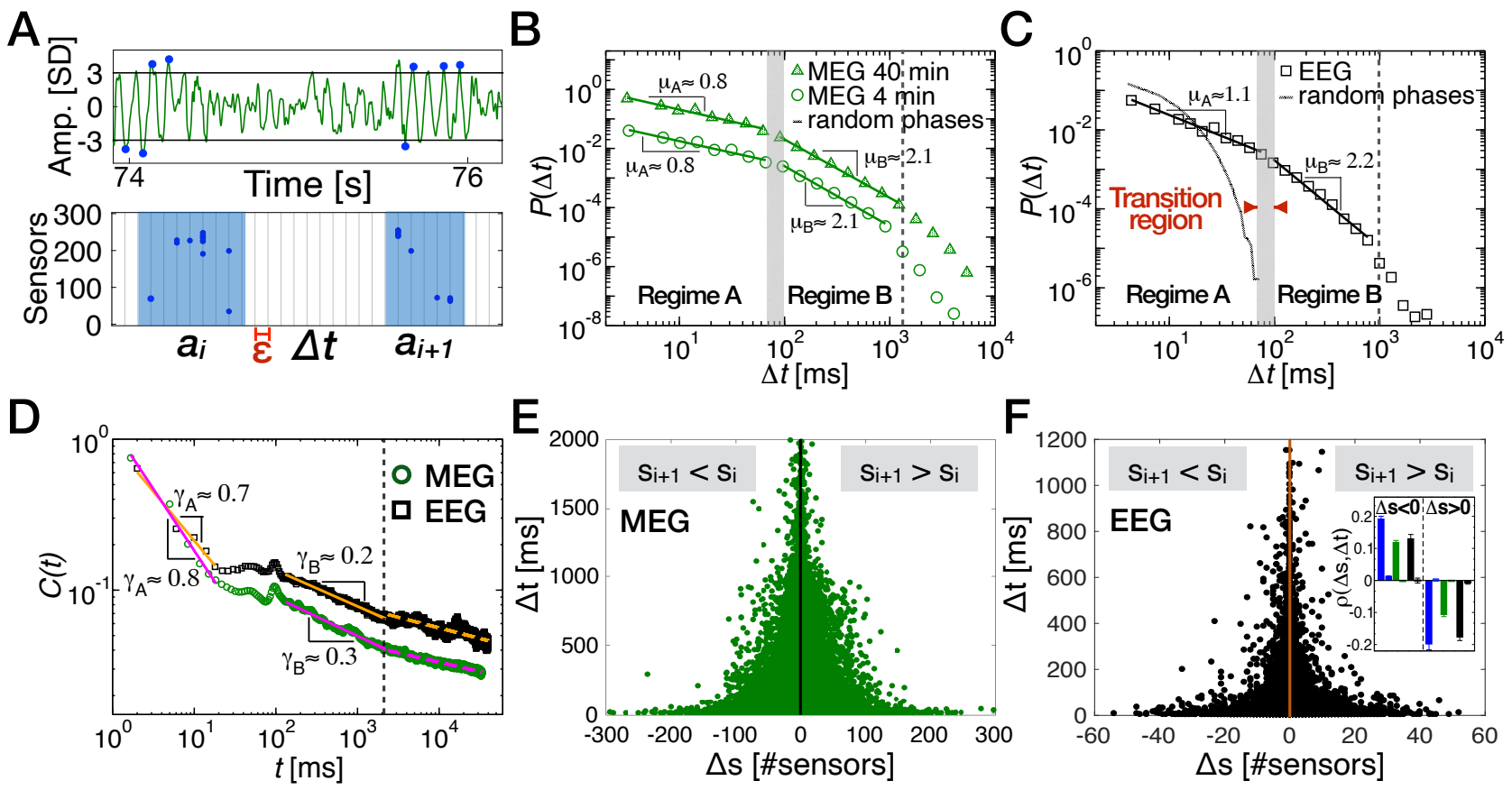

Fig. 1: The resting-state cascading process is characterized by a crossover in the dynamics occurring around $100 \mathrm{~ms}$, the characteristic time of the alpha rhythm. (A) (Top panel) MEG signal of resting-state activity of the human brain. The most extreme point in each excursion beyond a threshold $h$ (horizontal black lines) is treated as a discrete event (blue dots). (Bottom panel) Raster of discrete events across all MEG sensors ( $\mathrm{n}=273$ ) over an approximately 60-ms segment of recording (single subject). An avalanche $a_{i}$ is defined as a sequence of temporal windows $\epsilon\left[\epsilon=2 T_{m e g}=3.3 \mathrm{~ms}\right.$ for the MEG (Materials and Methods) $]$ with at least one event in any of the sensors, preceded and followed by at least one window with no events in any of the sensors. The same procedure is used for the EEG $\left(\epsilon=T_{e e g}=4 \mathrm{~ms}\right)$. (B) Distributions of quiet times $P(\Delta t)$ between consecutive avalanches in 4-min (green circle) and 40-min (green triangles) MEG recordings. Distributions show a double power-law behavior with a regime (A) for short $\Delta t$ 's characterized by an exponent $\mu_{A} \simeq 0.8$ (4-min data: $0.7848 \pm 0.0620 ; 40$-min data: $0.8103 \pm 0.0606$ ), and a regime (B) for long $\Delta t$ 's exhibiting an exponent $\mu_{B} \simeq 2$ (4-min data: $2.0329 \pm 0.0724$; 40-min data: $1.9766 \pm 0.0400$ ). The transition region (shaded area) between regime $(\mathrm{A})$ and $(\mathrm{B})$ is located around $100 \mathrm{~ms}$. Quiet time distributions from surrogate data (Materials and Methods) show an exponential behavior (black curve). (C) The distribution of quiet times in EEG recordings of the awake resting-state exhibits a double power-law behavior with a regime (A) for short $\Delta t$ 's characterized by an exponent $\mu_{A} \simeq 1.1(1.0696 \pm 0.2801)$, and a regime (B) for long $\Delta t$ 's with an exponent $\mu_{B} \simeq 2.2(2.2006 \pm 0.0839)$. The transition region (shaded area) between regimes (A) and (B) is located at around $100 \mathrm{~ms}$, as in the MEG. Quiet time distributions from surrogate data (Materials and Methods) show an exponential behavior (black curve). (D) Auto-correlation $C(t)$ of the activity measured in each time bin $\epsilon$ as the number of active sensors (i.e. absolute value of the signal larger than $h$ ). In the short time scales corresponding to regime $(\mathrm{A}), C(t)$ decays as a power-law with an exponent $\gamma_{A}$ (MEG: $\gamma_{A}=0.7633 \pm 0.0540$; EEG: $\left.\gamma_{A}=0.6627 \pm 0.0990\right)$. After the transition region between $20 \mathrm{~ms}$ and $120 \mathrm{~ms}$ in which $C(t)$ is nearly constant showing a small peak at $t=100 \mathrm{~ms}$, the autocorrelation follows a power-law with an exponent $\gamma_{B}$ (MEG: $\gamma_{B}=0.2480 \pm 0.0014 ;$ EEG: $\left.\gamma_{B}=0.2348 \pm 0.0016\right)$. (E) Scatter plot between $\Delta s$ and $\Delta t$ for all MEG subjects. Negative $\Delta s$ 's are positively correlated with their corresponding quiet times, whereas positive $\Delta s$ 's are anti-correlated with their relative quiet times (Spearman's correlation coefficient: $\rho(\Delta s<0, \Delta t)=0.1916 \pm 0.0088$ and $\rho(\Delta s<0, \Delta t)=0.1188 \pm 0.0061$ in 40-min and 4-min MEG recordings, respectively; $\rho(\Delta s>0, \Delta t)=-0.1064 \pm 0.0065$ in 4-min MEG, $\rho(\Delta s>0, \Delta t)=-0.1985 \pm 0.0181$ in 40-min MEG). (F) The scatter plot between $\Delta s$ and $\Delta t$ for all EEG subjects exhibits the same behavior observed in the MEG (Spearman's correlation coefficient: $\rho(\Delta s<0, \Delta t)=0.1301 \pm 0.0135 ; \rho(\Delta s>0, \Delta t)=-0.1765 \pm 0.0111)$. Inset: Spearman's correlation coefficient $\rho(\Delta s, \Delta t)$ calculated separately for $\Delta s<0$ and $\Delta s>0$ (Blue: MEG 40 min; Green: MEG 4 min; Black: EEG). $\rho(\Delta s, \Delta t)$ values obtained for avalanche time series obtained from surrogate data (Materials and Methods) are plotted next to each bar, and are very close to zero in all cases. $t$-tests comparing original and surrogate data show that detected correlations between $\Delta s$ and $\Delta t$ are significant $(p$-value $<0.001)$.

The correlation properties of the cascading process can be related to power-law exponents of the quiet time distribution [53]. Thus, the crossover between distinct power-law regimes indicates a change in the cascading dynamics at the temporal scale of the alpha rhythm. In Fig. 1DD, we show the autocorrelation function $C(t)$ of the quantity $n(t)$, the number of events occurring per unit time during the cascading process. We note that $n(t)=0$ during the quiet times, which corresponds to periods with no activity (Fig. 1A). We find that $C(t)$ exhibits two distinct power-law regimes for $t<1 \mathrm{~s}$, 
separated by a transition region located between $20 \mathrm{~ms}$ and $120 \mathrm{~ms}$. At short time scales [regime (A)], $C(t)$ decays as a power-law for both MEG and EEG with an exponent $\gamma_{A} \simeq 0.8$; for $120<t<1,000$ ms [regime (B)], $C(t)$ follows a power-law with a smaller exponent $\gamma_{B} \simeq 0.3$ (Fig. 11D). The crossover in $C(t)$ parallels the crossover observed in the quiet time distributions for $\Delta t<1 \mathrm{~s}$ (Fig. 1 $\mathrm{B}, \mathrm{C}$ ), and indicates that the correlations in the cascading process strongly depend on the time scales. We observe that $C(t)$ exhibits a further transition to a slower decaying regime around $t=1 \mathrm{~s}$, which in turn parallels the cutoff observed in $P(\Delta t)$ (Fig. 1 $1 \mathrm{~B}, \mathrm{C}$ ).

The observed functional behavior of $P(\Delta t)$, as well as the autocorrelation function $C(t)$, suggests that the relationship between consecutive avalanches (and thus the underlying collective neural dynamics) undergoes a transition around $\Delta t \simeq 100 \mathrm{~ms}$. Here, we hypothesize that such a transition reflects a property of the neural dynamics associated with the generation and propagation of the alpha rhythm in resting-state brain activity. To verify this hypothesis, we investigate whether the collective neural dynamics encoded in avalanche characteristics (e.g. their sizes) exhibits signatures of dynamic transitions that correlate with alpha oscillations.

\subsection{The cascading process in the awake resting-state follows an attenuation- amplification dynamics (AAD)}

To this end, we consider the avalanche size increments $\Delta s=s_{i+1}-s_{i}$ between the size of avalanche $a_{i}$ and the size of the subsequent avalanche $a_{i+1}$, and analyze their relationship with the corresponding quiet times $\Delta t_{i}$ (Fig. 1). Negative values of $\Delta s$ imply attenuation, i.e. the avalanche $a_{i+1}$ is smaller than its preceding one $a_{i}$, whereas positive values imply amplification, namely, the avalanche $a_{i+1}$ is larger than the preceding avalanche $a_{i}$

We first examine the scatter plot between $\Delta s$ 's and the corresponding $\Delta t$ 's (Figs. $1 \mathrm{E}, \mathrm{F}$ ). For $\Delta s<0$, we observe that large negative $\Delta s$ 's occur with short quiet times $\Delta t$ 's and vice versa, both in MEG (Fig. 1E) and EEG recordings (Fig. 1F). The corresponding value of the Spearman's correlation coefficient $\rho(\Delta s<0, \Delta t$ ) (Materials and Methods) is significantly larger than zero (Fig. 1 $\mathrm{F}$, inset), indicating that negative size increments are positively correlated with their corresponding quiet times, i.e. the more negative the increment, the shorter the quiet time separating two avalanches. In contrast, we find that positive size increments, $\Delta s>0$, tend to be significantly anti-correlated with the corresponding quiet times both in MEG and EEG data (Fig. 1F, inset).

To dissect the dependency of size increments on the time lag separating consecutive avalanches and identify the connection with the alpha rhythm, we scrutinize the correlation landscape hidden in the density distribution across the $\Delta t \Delta s$ plane of the scatter plots shown in Figs. $1 \mathrm{E}$ and $\mathrm{F}$. To this aim, we systematically compare the spatial structure of the density in the plane $\Delta t \Delta s$ with surrogate densities obtained by randomly reshuffling avalanche sizes. In this way, both the distribution and the temporal order of quiet times are preserved, as well as the size distribution (Fig. 2A).

The local density distribution resulting from the avalanche process can be described using the conditional probability $P\left(s_{0}, t_{0}\right) \equiv P\left(\Delta s<s_{0} \mid \Delta t<t_{0}\right)=N\left(\Delta s<s_{0} \mid \Delta t<t_{0}\right) / N\left(\Delta t<t_{0}\right)$, where the thresholds $s_{0}$ and $t_{0}$ delimit the plane region $R\left(s_{0}, t_{0}\right)$ under investigation (Fig. 2A A, left panel). An analogous quantity $P^{*}\left(s_{0}, t_{0}\right)$ can be associated with the surrogate region resulting from the size reshuffling procedure. The difference $\delta P\left(s_{0}, t_{0}\right) \equiv \delta P\left(\Delta s<s_{0} \mid \Delta t<t_{0}\right)=P\left(s_{0}, t_{0}\right)-P^{*}\left(s_{0}, t_{0}\right)$ is a measure of the likelihood that a specific distribution of points in the region $R\left(s_{0}, t_{0}\right)$ results from the actual avalanche dynamics, rather than from random avalanche occurrence, and is used to examine the relationship between sizes of consecutive avalanches as a function of the quiet times separating 

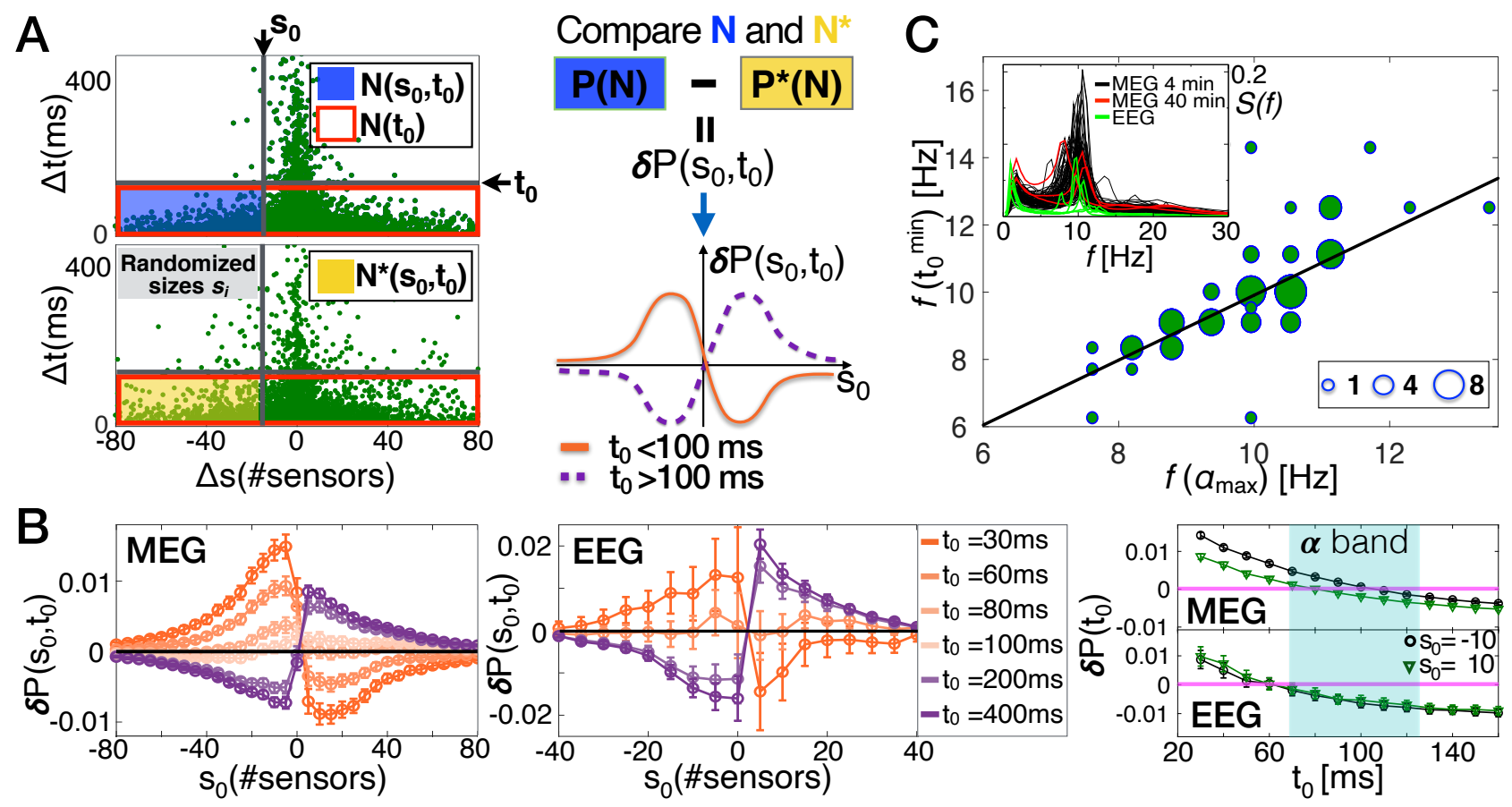

Fig. 2: The monotonic relationship between consecutive avalanches undergoes a transition from an attenuation to an amplification regime that correlates with the characteristic time of the alpha rhythm. (A) Schematic definition of the quantity $\delta P\left(s_{0}, t_{0}\right) \equiv \delta P\left(\Delta s<s_{0} \mid \Delta t<t_{0}\right)$. (Left panel) The conditional probabilities $P\left(s_{0}, t_{0}\right)$ and the surrogate conditional probabilities $P^{*}\left(s_{0}, t_{0}\right)$ are proportional to the number of points $N\left(s_{0}, t_{0}\right)$ and $N^{*}\left(s_{0}, t_{0}\right)$ in the region $R\left(s_{0}, t_{0}\right)$ of the plane $\Delta s \Delta t$ defined by the thresholds $s_{0}$ and $t_{0}$ (blue and yellow rectangles, respectively). (Right panel, top) The quantity $\delta P\left(s_{0}, t_{0}\right)$ compares the original and the surrogate density in the region $R\left(s_{0}, t_{0}\right)$ of the plane, and is defined as the difference between $P\left(s_{0}, t_{0}\right)$ and $P^{*}\left(s_{0}, t_{0}\right)$, the conditional probabilities associated with the density $N$ and $N^{*}$ for original and surrogate data (Materials and Methods). (Right panel, bottom) The quantity $\delta P\left(s_{0}, t_{0}\right)$ as a function of $s_{0}$ for a given threshold $t_{0}$ on $\Delta t$ 's exhibits two relevant scenarios: For $t_{0}<100$ ms (attenuation regime, orange thick line), $\delta P\left(s_{0}, t_{0}\right)$ has a maximum for $s_{0}<0$, implying that $s_{i+1}$ tends to be smaller than $s_{i}$ for $\Delta t<t_{0}$; for $t_{0}>100 \mathrm{~ms}$ (amplification regime, purple dashed line), $\delta P\left(s_{0}, t_{0}\right)$ has a maximum for $s_{0}>0$, implying that, for $\Delta t<t_{0}, s_{i+1}$ tends to be larger than $s_{i}$. (B) $\delta P\left(s_{0}, t_{0}\right)$ as a function of the threshold $s_{0}$ on $\Delta s$ for different values of the threshold $t_{0}$ on $\Delta t$ 's for MEG (left panel) and EEG (middle panel) data. The error bar on each data point is two times the standard deviation $\sigma^{*}$ associated with the surrogates $P^{*}\left(s_{0}, t_{0}\right)$ (see Materials and Methods). For a given $t_{0}$, the maximum in $\delta P\left(s_{0}, t_{0}\right)$ indicates the preferred relation between consecutive avalanches separated by quiet times shorter than $t_{0}$. Both in MEG $(4 \mathrm{~min})$ and EEG recordings (left and middle panel), for $t_{0}$ smaller than $\approx 100 \mathrm{~ms}, \delta P\left(s_{0}, t_{0}\right)$ has a maximum at $s_{0}<0$, and thus an avalanche tends to be smaller than its preceding one $\left(s_{i+1}<s_{i}\right.$, attenuation regime). On the contrary, for $t_{0}$ larger than $\approx 100 \mathrm{~ms}$, the maximum moves towards positive $s_{0}$, implying that a given avalanche tends to be larger than the preceding one $\left(s_{i+1}>s_{i}\right.$, amplification regime). For $t_{0} \approx 100 \mathrm{~ms}, \delta P\left(s_{0}, t_{0}\right)$ is very close to zero for each $s_{0}$, indicating that at $t_{0} \simeq 100 \mathrm{~ms}$, there is not a preferred sign for $\Delta s$, and that $\Delta t=100 \mathrm{~ms}$ is a transition point from one dynamical regime to another - an attenuation-amplification transition. A similar behavior is observed in $40 \mathrm{~min}$ MEG recordings (Fig. S2). (Right panel) $\delta P\left(s_{0}, t_{0}\right)$ as a function of $t_{0}$ for $s_{0}=-10$ and $s_{0}=10$ in MEG (4 min) and EEG. For each $s_{0}, \delta P\left(t_{0}\right)$ transitions from positive to negative values in a range of $t_{0}$ corresponding to the alpha band $(\approx 80 \mathrm{~ms}$ and $\approx 130 \mathrm{~ms})$. A similar behavior is observed in 40 min MEG (Fig. S2). (C) The scatter plot between $f\left(\alpha_{\max }\right)$ and $f\left(t_{0}^{\min }\right)$ shows that the attenuation-amplification transition in $\delta P\left(s_{0}, t_{0}\right)$ correlates with the maximum in the $\alpha$ band of the power spectrum. The size of each circle is proportional to the number of points (subjects) in the corresponding region of the plane. The black thick line is a linear fit, $Y=A \cdot X+B$, with $A=0.96$ and $B=0.26\left(r^{2}=0.80\right)$. Inset: Power spectrum $S(f)$ for each subject (black lines, MEG 4 min; red lines, MEG 40 min; green lines, EEG).

them. We indicate with $\sigma^{*}$ the standard deviation associated with the surrogates $P^{*}\left(s_{0}, t_{0}\right)$. Thus, if $\left|\delta P\left(s_{0}, t_{0}\right)\right|>2 \sigma^{*}, P\left(s_{0}, t_{0}\right)$ and $P^{*}\left(s_{0}, t_{0}\right)$ are significantly different ( $p$-value $<0.05$; see Materials and Methods), and the distribution of points in the region $R\left(s_{0}, t_{0}\right)$ is considered to reflect a specific, non-random relationship between $\Delta s$ and $\Delta t$.

We study $\delta P\left(s_{0}, t_{0}\right)$ as a function of $s_{0}$ for fixed $t_{0}$ values. For each fixed value of $t_{0}$, there are two possible relevant scenarios for the function $\delta P\left(s_{0}, t_{0}\right)$ (Fig. 2A, right panel): (i) Attenuation regime. $\delta P\left(s_{0}, t_{0}\right)$ is positive and monotonically increases for $-\infty<s_{0}<0$, reaches a maximum, and then 
decreases, taking a minimum at some $s_{0}>0$ (Fig. 22A, right bottom panel, orange thick line). In this regime, the size increments, $\Delta s=s_{i+1}-s_{i}$, tend to be negative, and the avalanche $a_{i+1}$ tends to be smaller than the preceding avalanche $a_{i}$; (ii) Amplification regime. $\delta P\left(s_{0}, t_{0}\right)$ is negative and monotonically decreases for $-\infty<s_{0}<0$, reaches a minimum, and then increases, taking a local maximum at some $s_{0}>0$ (Fig. 2A, right bottom panel, purple dashed line). In the latter regime, the amplification regime, the size increments tend to be positive, and a given avalanche $a_{i+1}$ tends to be larger than its preceding avalanche $a_{i}$ (Fig. 2A, right panel, purple dashed line). Following this approach, we show that the attenuation and amplification regimes correspond to regimes (A) and (B) defined in Fig. 1 .

We first analyze $\delta P\left(s_{0}, t_{0}\right)$ as a function of $s_{0}$ for several fixed values of the threshold $t_{0}$ (Fig. $2 \mathrm{~B}$ ). If the relation between consecutive avalanches depends on their distance in time, then $\delta P\left(s_{0} ; t_{0}\right)$ changes as we consider different $t_{0}$ values. In Fig. $2 \mathrm{~B}$ we show the function $\delta P\left(s_{0}, t_{0}\right)$ evaluated for $t_{0}$ values ranging between $30 \mathrm{~ms}$ and $1000 \mathrm{~ms}$. We find that $\delta P\left(s_{0} ; t_{0}\right)$ follows the attenuation regime for $t_{0}$ 's smaller than approximately $100 \mathrm{~ms}$, whereas it conforms to the amplification regime for larger $t_{0}$ values. Thus, size increments $\Delta s$ tend to be negative if the quiet time between consecutive avalanches is shorter than $100 \mathrm{~ms}$, and positive otherwise. This behavior is also observed in 40-min MEG data (Fig. S2), and is consistent across subjects (Fig. S3). The tendency for negative $\Delta s$ 's to be coupled with $\Delta t<100 \mathrm{~ms}$ implies that avalanche sizes preferentially exhibit a decreasing trend on short time scales, a clear sign of attenuated bursting activity. On the other hand, the significant likelihood for positive increments at longer time scales suggests the presence of a regulatory mechanism that, after discharging cycles with decreasing avalanche sizes, amplifies bursting activity and leads to the appearance of larger avalanches. The transition from the attenuation to the amplification regime occurs at $t_{0} \approx 100 \mathrm{~ms}$, where we observe that $\delta P\left(s_{0}, t_{0}\right) \simeq 0$ (Fig. $2 \mathrm{~B}$ ). Importantly, $\delta P\left(s_{0}, t_{0}\right) \simeq 0$ implies that $P\left(s_{0}, t_{0}\right) \simeq P^{*}\left(s_{0}, t_{0}\right)$, which in turn implies that, in the plane $\Delta t \Delta s$, the local density around $\Delta t \approx 100 \mathrm{~ms}$ is comparable to the density obtained with the reshuffled avalanche sizes. Crucially, we observe that the attenuation and amplification regimes correspond to regime (A) and (B) in the quiet time distributions (Fig. 1 B B,C), and the attenuation-amplification transition coincides with the crossover from regime $(\mathrm{A})$ to regime $(\mathrm{B})$ (Fig. 1B-D).

\subsection{The AAD of the awake resting-state correlates with the alpha rhythm}

To identify the transition point $t_{0}^{\min }$ from the attenuation to the amplification regime, we study $\delta P\left(\Delta s<s_{0}, \Delta t<t_{0}\right)$ as a function of $t_{0}$ for a range of fixed $s_{0}$ values - i.e. $\delta P\left(t_{0}\right)$ (Fig. $2 \mathrm{~B}$, right panel). We plot $\delta P\left(t_{0}\right)$ for $s_{0}= \pm 10$, where the quantity $\delta P\left(\Delta s<s_{0}, \Delta t<t_{0}\right)$ is generally non-zero away from the transition point (Fig. 2, right and middle panels). A similar behavior is observed for other $s_{0}$ values (Fig. S2). We find that the transition from positive to negative $\delta P\left(t_{0}\right)$ lies mostly in the range [70 ms, $130 \mathrm{~ms}$ ] (Fig. 2 and $\mathrm{S} 2$ ), which approximately corresponds to oscillations in the frequency range [8-13] Hz, commonly identified as the alpha band. Similar results are obtained in 40min MEG recordings (Fig. S2). Then we define $t_{0}^{\min }$ as the $t_{0}$ that minimizes $\left|\delta P\left(\Delta s<s_{0} \mid \Delta t<t_{0}\right)\right|$ over a range of relevant $s_{0}$ values, namely $t_{0}^{\min }=\min _{t_{0}}\left(\sum_{s_{0}=-30}^{30}\left|\delta P\left(\Delta s<s_{0} \mid \Delta t<t_{0}\right)\right|\right)$.

Next, we show that the attenuation-amplification transition correlates with the timescale characteristic of the alpha rhythm, and is consistent across subjects in both MEG and EEG recordings of the awake resting-state (Fig. S3). In Fig. 2 C, for all subjects, we plot the frequency $f\left(\alpha_{\max }\right)$, which corresponds to the maximum power in the alpha band, versus the transition frequency $f\left(t_{0}^{\min }\right)=1 / t_{0}^{\min }$ corresponding to the transition point $t_{0}^{\min }$ from the attenuation regime to the amplification regime 
(Fig. 2 2 ). We observe that the frequency $f\left(t_{0}^{\min }\right)$ correlates with $f\left(\alpha_{\max }\right)\left(r^{2}=0.80\right)$, indicating that the attenuation-amplification transition is intimately connected to the dynamics of alpha oscillations. Importantly, the correlation between the transition frequency $f\left(t_{0}^{\min }\right)$ and the $f\left(\alpha_{\max }\right)$ suggests that the crossover both in the distribution of quiet times and in the quantity $\delta P\left(s_{0}, t_{0}\right)$ may relate to a basic difference in the cascading process within and between alpha cycles.

\subsection{The alpha "waxing and waning" is a long-term attenuation-amplification mechanism that obeys the Omori law}

We have shown that the $\mathrm{AAD}$ of the resting-state cascading process acts on a timescale of less than a second, with an attenuation-amplification transition that correlates with the characteristic frequency of alpha oscillations. Importantly, alpha waves are also known to exhibit long-term alternation between higher and lower amplitude fluctuations over the timescales of several seconds - the so called "waxing and waning" phenomenon [41, 40, 19]. To characterize the resting-state cascading process in relation to alpha "waxing and waning", we focus on large cascades of activity, which we define as avalanches larger than a given size $s^{*}$ and label them as main avalanches, $A^{*}$ (Fig. 3A, left top panel). Large avalanches are synchronous events consisting of time-clustered, higher amplitude signal fluctuations over a large number of sensors - see the raster plot in Fig. 3A, left bottom panel. We observe that, following a main avalanche $A^{*}$ (identified by blue arrows in the right panel of Fig. $3 \mathrm{~A}$ ), the sizes of activity cascades tend to rapidly decrease, with fluctuations that also decrease with the time elapsed from $A^{*}$. We refer to the activity following a main avalanche as the Omori sequence (Fig. 3A, left panel).

Such dynamics follow the attenuation-amplification principle over timescales ranging between a few seconds to a few tens of seconds, and is strongly reminiscent of the alpha "waxing and waning" [40]. Following this analogy, we analyze the brain activity around the main avalanches $A^{*}$ 's. In Fig. 3A (right panel), we show the analysis of a raster plot segment from an Omori sequence (marked in magenta in the left panel). We observe that the main avalanche identified in the raster plot (top panel) corresponds to higher amplitude alpha bursts (middle panel), with large peaks in the alpha power $S(\alpha)$ (bottom panel). Furthermore, we notice that before and after the main avalanche, both the signal amplitude and the alpha power decrease considerably (Fig. $3 \mathrm{~A}$, middle and bottom panels).

Next, we proceed to quantify the Omori sequences by analyzing the number $N(t)$ of avalanches per unit time occurring after a time $t$ has elapsed from the main avalanches. We find that $N(t)$ decays as $t^{-p}$ across a wide range of $t$ 's in both 4-min MEG and EEG recordings (Fig. 3B). We observe that the exponent $p$ is close to one for a range of threshold values $s^{*}$ used to identify the main avalanches, and its value decreases when the threshold $s^{*}$ becomes too large (Fig. $3 \mathrm{~B}$, insets). This behavior is also observed in 40-min MEG recordings (Fig. S4), and is consistent across subjects (Fig. S4). The power-law decay $N(t) \propto t^{-p}$ of the number of events following a main avalanche is consistent with the generalized Omori law, $N(t) \propto(t+c)^{-p}$ (where $c$ is a parameter related to the onset of the power-law regime), describing the temporal organization of aftershocks following a main earthquake [63, 47, 12, and indicates that large avalanches somehow trigger the ones that follow. Moreover, we observe that the power-law cutoff is located around $5 \mathrm{~s}$ (Fig. 3 $3 \mathrm{~B}$ ). This indicates that the activity after a large cascade is characterized by temporal clustering over unusually long timescales which correspond to the typical timescales of the "waxing and waning" [41, 40, 19].

Importantly, we find that the AAD is a constituent part of the resting-state Omori-type dynamicswhich in this context acts as a long-term attenuation-amplification mechanism. Indeed, we observe 
bioRxiv preprint doi: https://doi.org/10.1101/2022.03.03.482657; this version posted March 4, 2022. The copyright holder for this preprint (which was not certified by peer review) is the author/funder, who has granted bioRxiv a license to display the preprint in perpetuity. It is made available under aCC-BY-NC-ND 4.0 International license.
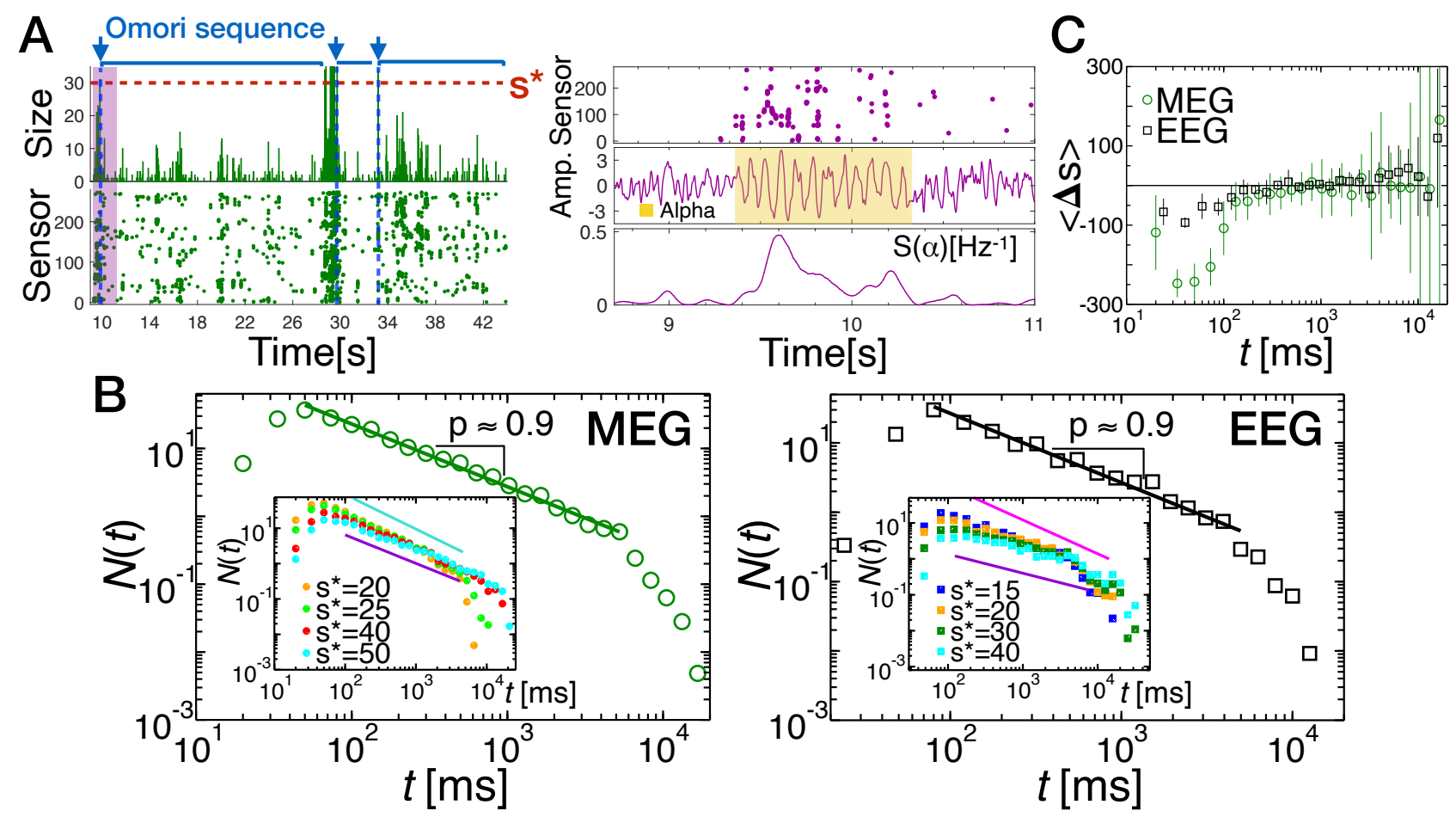

Fig. 3: The alpha "waxing and waning" is described by the Omori law in the underlying neural cascading process. (A) (Left panel) A raster plot of $35 \mathrm{~s}$ of activity (bottom) and the corresponding avalanche sizes (top) showing three Omori sequences of different durations ranging between about $2 \mathrm{~s}$ and $15 \mathrm{~s}$ and including several alpha cycles. Each sequence starts with a main avalanche $A^{*}$ with a size larger than 30 (blue arrow). Activity decreases over time until the next main avalanche. (Right panel, top) Raster plot of the start of the first Omori sequence highlighted in magenta in the left panel. Brain activity around the main avalanche corresponds to high-amplitude alpha bursts (middle panel, yellow), preceded and followed by lower-amplitude fluctuations. (Bottom) The instantaneous power at $10 \mathrm{~Hz}$ peaks around the main avalanche, and then rapidly decreases. (B) (left panel ) $N(t)$ as a function of the time $t$ elapsed after a main avalanche $A^{*}$ in 4-min MEG resting brain activity. $N(t)$ decreases as the reciprocal of the time elapsed from the main avalanche $A^{*}$, i.e. $N(t) \propto t^{-p}$ with $p \simeq 1\left(p=0.9240 \pm 0.0169 . s^{*}=30 ; s * /(\# M\right.$ EGsensors $\left.) \simeq 0.11\right)$. Inset: $N(t)$ for different values of the threshold $s^{*}$ defining a main avalanche $A^{*}$. The exponent $p$ describing the power-law decay of $N(t)$ ranges between $0.9773 \pm 0.0302$ (turquoise line) and $0.8054 \pm 0.020$ (violet line). Similar results are obtained in 40-min MEG recordings (Fig. S4). (Right panel) The Omori-law is verified in the EEG of the resting state, with $p=0.9384 \pm 0.0321$ for $s^{*}=10\left(s^{*} /(\#\right.$ EEGsensor $\left.s) \simeq 0.16\right)$. Inset: $N(t)$ for different values of the threshold $s^{*}$ defining a main avalanche $A^{*}$. The exponent $p$ describing the power-law decay of $N(t)$ ranges between $0.9384 \pm 0.0321$ (magenta line) and $0.5289 \pm 0.0471$ (violet line). (C) Average $\Delta s=s_{i+1}-s_{i}$ between consecutive avalanches occurring within two main avalanches as a function of the time $t$ elapsed from the first main avalanche.

that, within an Omori sequence, the average $\Delta s$ between consecutive avalanches is always negative for $t<100 \mathrm{~ms}$, and transitions to positive values between a few hundreds of milliseconds to a few seconds (Fig. $3 \mathrm{C}$ ), in line with the AAD described in the previous section (Fig. 2).

\subsection{The cascading process during sleep does not show AAD and does not obey the Omori law}

We have demonstrated that collective neural activity during resting wakefulness is characterized by $\mathrm{AAD}$, namely, the alternation of two distinct dynamical regimes: an attenuation regime within the alpha cycle, and an amplification regime between alpha cycles (Fig. 2). The AAD is part of the Omori-type dynamics encompassing resting-state brain activity over timescales of several seconds. To verify that these are specific characteristics of the cascading dynamics in the awake resting-state dominated by the alpha rhythm, we next investigate the dynamics of neural cascades during nonrapid eye movement (NREM) sleep. In contrast to the awake resting-state, during NREM sleep, brain activity does not show alpha oscillations, but is largely dominated by slow oscillations in the 

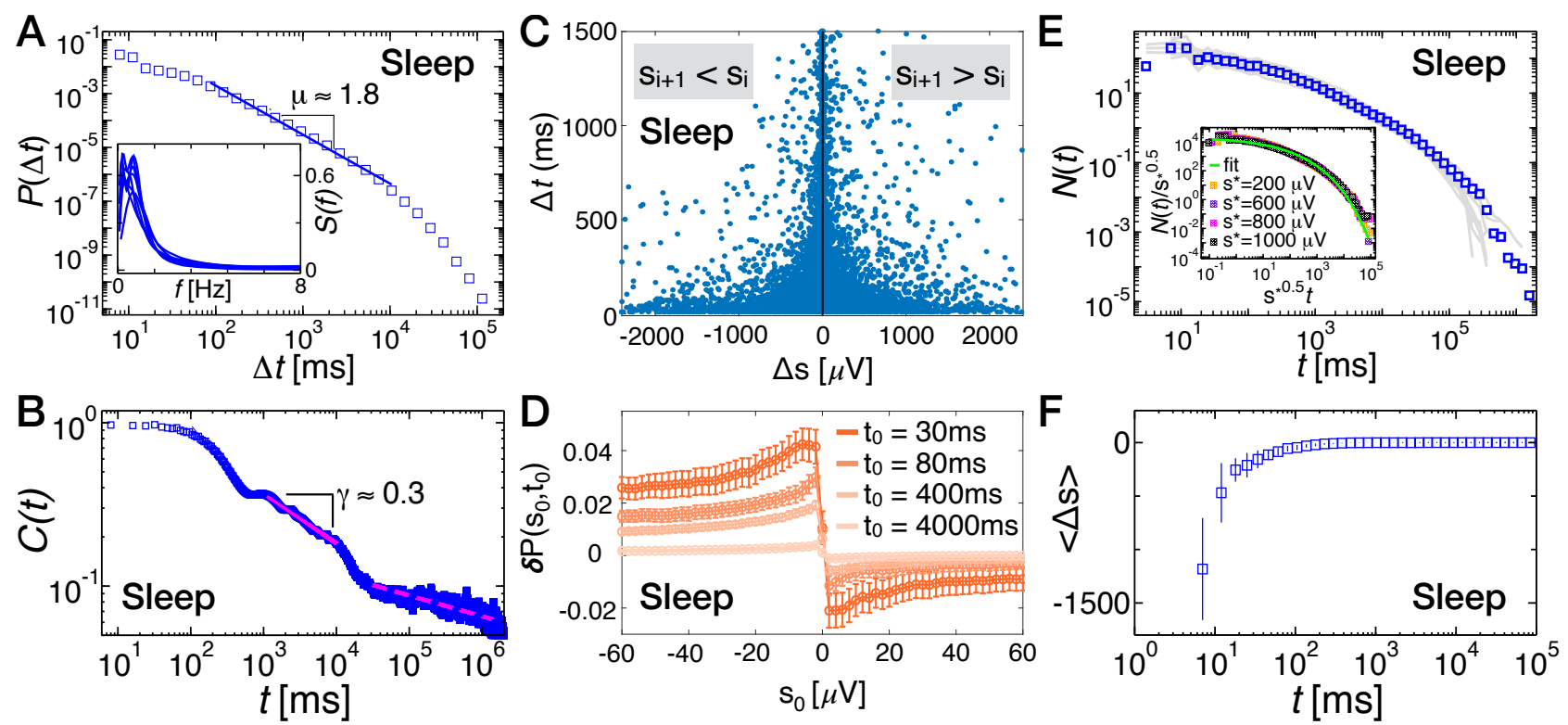

Fig. 4: The cascading process during sleep does not exhibit AAD and does not obey the Omori law. (A) Distributions of quiet times $P(\Delta t)$ between consecutive avalanches during sleep (pooled data). Unlike the resting state, during sleep, quiet time distributions show a single power-law regime characterized by an exponent $\mu=1.8191 \pm 0.0392$ and followed by an exponential cutoff. Inset: Power spectra for individual subjects. The maximum is always in the range $0.5-2 \mathrm{~Hz}$, within the delta band. (B) Auto-correlation $C(t)$ of the instantaneous activity measured in each time bin $\epsilon$. For $t<500 \mathrm{~ms}, C(t)$ exhibits an exponential decay, which is followed by a plateau between $500 \mathrm{~ms}$ and 1,000 $\mathrm{ms}$, corresponding to $1-2 \mathrm{~Hz}$ delta oscillations. For $10^{3}<t<10^{4} \mathrm{~ms}, C(t)$ decays as a power-law with an exponent $\gamma=0.3017 \pm 0.0011$. (C) Scatter plot between $\Delta s$ and $\Delta t$ during sleep (all subjects). Negative $\Delta s$ 's are positively correlated with their corresponding quiet times, whereas positive $\Delta s$ 's are anti-correlated with their relative quiet times. The Spearman's correlation coefficient $\rho(\Delta s, \Delta t)$ calculated separately for $\Delta s<0$ and $\Delta s>0$. $\rho(\Delta s, \Delta t)$ is positive for $\Delta s<0$ and negative for $\Delta s>0: \rho(\Delta s<0, \Delta t)=0.1627 \pm 0.0032 ; \rho(\Delta s>0, \Delta t)=-0.1022 \pm 0.0041$. (D) The quantity $\delta P\left(s_{0}, t_{0}\right)$ as a function of $s_{0}$ for different values of the threshold $t_{0}$ on $\Delta t$ 's. The error bar on each data point is $2 \sigma^{*}$ ( $\sigma^{*}$ is the $\mathrm{SD}$ associated with the surrogates $P^{*}$ (Materials and Methods). For each value of $t_{0}, \delta P$ is always positive for $s_{0}<0$ and negative for $s_{0}>0$, and takes its maximum (minimum) at $s_{0} \simeq-10\left(s_{0} \simeq 10\right)$. Hence, given a couple of avalanches, the second avalanche tends to be smaller than the first one (attenuation regime), independently of the quiet time separating them (cf. Fig. 2). (E) Number of avalanches per unit time, $N(t)$, occurring after a main avalanche, i.e. an avalanche of size larger than $s^{*}$ (Grey lines: Individual subjects; Symbols: Pooled data). Unlike the resting wake, $N(t)$ does not obey the Omori law, but is well fitted by a stretched exponential $N(t) \propto e^{-(t / \tau)^{\beta}}$. Inset: $N(t)$ for different values of the threshold $s^{*}$ used to define a main avalanche. Data for different $s^{*}$ 's collapse onto a single curve when $t$ is rescaled by $s^{*^{0.5}}$. Green line: Stretched exponential fit $N(t) \propto e^{-(t / \tau)^{\beta}}$, with $\beta=0.25$. (F) The average $\Delta s=s_{i+1}-s_{i}$ between consecutive avalanches occurring within two main avalanches is always negative, and monotonically increases with $t$, the time elapsed from the main avalanche.

delta band $(1-4 \mathrm{~Hz})[50]$.

We consider EEG recordings across approximately $8 \mathrm{~h}$ of night sleep (Materials and Methods), and analyze the distribution of quiet times between consecutive avalanches. We observe that, unlike the awake resting-state, the distribution of quiet times during NREM sleep exhibits a single power-law regime with an exponent $\mu_{s} \simeq 1.8(1.8734 \pm 0.0465)$, followed by an exponential decay for $\Delta t>10 \mathrm{~s}$ (Fig. 4A). Correspondingly, the autocorrelation function $C(t)$ of the instantaneous activity shows a power-law decay with an exponent $\gamma \simeq 0.3$ for $1 \mathrm{~s}<t<10 \mathrm{~s}$, followed by a slower decay for larger $t$ 's (Fig. 4B). Unlike in the awake resting-state (Fig. 1), during sleep, $C(t)$ exhibits an exponential decay at shorter timescales $(t<500 \mathrm{~ms})$ (Fig. $4 \mathrm{~B}$ ).

Next, we examine the scatter plot between the $\Delta s$ 's and the corresponding $\Delta t$ 's (Fig. $4 \mathrm{C}$ ). We find that large negative $\Delta s$ 's occur with short quiet times $\Delta t$ 's, while positive size increments tend to be anti-correlated with the corresponding quiet times (Fig. $4 \mathrm{C}$ ). This behavior is similar to the behavior we observed during resting wakefulness (Figs. 1 $\mathrm{E}, \mathrm{F}$ ).

However, the relationship between consecutive avalanches as a function of the time separation 
exhibits a rather different behavior during NREM sleep. We analyze the quantity $\delta P\left(s_{0} ; t_{0}\right)$ defined in Fig. 2 as a function of $s_{0}$ for a range of $t_{0}$ values between $30 \mathrm{~ms}$ and 4,000 ms (Fig. 4D). We find that $\delta P\left(s_{0} ; t_{0}\right)$ follows the attenuation regime defined in Fig. 2 for all $t_{0}$ values (Fig. $4 \mathrm{D}$ ), namely, the size increments $\Delta s$ between consecutive avalanches tend to be always negative. This implies that avalanche sizes preferentially exhibit a decreasing trend, an attenuation effect that is particularly strong for $\Delta t$ 's shorter than $400 \mathrm{~ms}$. Such a behavior is in stark contrast with our observations during resting wakefulness, where we found a transition from an attenuation regime $-\Delta s<0$ for $\Delta t<100$ ms - to an amplification regime $-\Delta s>0$ for $\Delta t>100 \mathrm{~ms}$ - at the characteristic time of the alpha rhythm (Fig. 2).

We next analyze the number $N(t)$ of avalanches per unit time occurring after a time $t$ has elapsed from a main avalanche $A^{*}$. We find that, unlike in the awake resting-state, $N(t)$ decays according to a stretched exponential, that is $N(t) \propto e^{-(t / \tau)^{\beta}}$ with $\beta=0.25$. This behavior is consistent across subjects (Fig. 4 $4 \mathrm{E}$, grey curves), and does not depend on the threshold $s^{*}$ used to define the main avalanches $A^{*}$, as demonstrated by the data collapse in the inset of Fig. 4 $4 \mathrm{E}$. Furthermore, in contrast to the awake resting-state, we find that the average $\Delta s$ in the sequence of avalanches between two main shocks is always negative, and slowly approaches zero for $t>(1-2 \mathrm{~s}$ ) (Fig. $4 \mathrm{~F}$ ).

\section{Discussion}

In this paper, we provided a description of the resting-state brain activity that uncovers the dynamic organization of neural activity cascades underlying brain oscillations, unifying two complementary approaches to neural synchronization — neuronal avalanches and oscillations [8, 22, 32, 39, 30, 34]. Our analysis shows that the collective neural dynamics underlying resting-state brain activity is governed by attenuation-amplification principles across timescales - which we link with prominent features of the alpha oscillations. On a timescale of a few alpha cycles $(<1 \mathrm{~s})$, the attenuation dynamics characterize the cascading process within the typical length of the alpha cycle, i.e. about $100 \mathrm{~ms}$ [7, 8], while the amplification dynamics occur over timescales of a few hundreds of milliseconds. Significantly, the attenuation-amplification transition consistently correlates with the dominant frequency in the alpha band. We have shown that these short-term attenuation-amplification dynamics (AAD) are part of a large-scale, size-dependent temporal structure of neural cascades that obeys the Omori law [63]: large avalanches (main avalanches) are followed by increasingly smaller avalanches at a rate that decays as a power-law of the time elapsed from the main avalanche - a long-term AAD-regulating brain activity over the timescale of seconds. Importantly, both the short-term AAD and the Omori law are unique to the awake resting-state, and are not present during sleep.

The dynamic structure of neural cascades during the awake resting-state contains the fingerprints of two key characteristics that have long been associated with the alpha rhythm: (i) The inhibitory effects in information processing, and (ii) The periodic fluctuations in amplitude known as "waxing and waning" [41, 66, 40, 11, 46, 21, 19]. The short-term AAD reported in the present study indicates that inhibition may be activated to gradually suppress the cascading process within about $100 \mathrm{~ms}$ during the attenuation regime, while excitability is successively enhanced to amplify neural cascades over the timescales of a few alpha cycles (a few hundreds of milliseconds). Coherently, at the crossover between attenuation and amplification, i.e. at about the $100 \mathrm{~ms}$ characteristic of alpha waves, there is no clear monotonic relation between consecutive avalanches, which is consistent with a random organization of avalanche sizes - a clear transition signature. This is consistent with the hypothesis that alpha- 
mediated reduction of information processing is applied in rhythmic cycles, a mechanism referred to as "pulsed inhibition" [25, 37]. At the same time, our findings also indicate an active role for alpha waves in timing the increase in network excitability. Furthermore, our analysis shows that the attenuationamplification principle governs resting-state brain activity across scales, revealing a precise structure in the cascading process underlying the long-term "waxing and waning" phenomenon [40. We found that the high-amplitude alpha bursts function as main inhibitory events with a characteristic time of a few seconds. Between such events - which we call main avalanches - smaller and increasingly sparser cascades occur, obeying the short-term AAD. These observations indicate that the precise short-term dual role - attenuating and amplifying - of the alpha rhythm is embedded in a size-time dependent long-term organization captured by the Omori law. The range of variability implied by the powerlaw behavior of the Omori law, previously known as "waxing and waning" phenomena with different timescales [40], may provide the brain with the flexibility necessary to organize complex streams of information, while maintaining precise information control through timely short-term AAD.

In stark contrast with our observations in the awake resting-state, we found that the cascading process during NREM sleep, where the alpha rhythm is absent, does not show AAD, and the avalanche occurrence rate after a large avalanche follows a stretched exponential decay. The stretched exponential can be understood as a superposition of exponential decays with different characteristic times. In this case, the cascading process can be seen as the superposition of many independently acting entities, each with a specific fixed cascading rate. Thus, the presence of a stretched exponential decay during sleep suggests that the corresponding emergent cortical patterns may depend on the multiple brain regions controlling sleep regulation, and on the coupling of different brain rhythms [26, 5, 52, 57, 29, 67, 2, 36]. This further confirms that the AAD and the Omori law are related to the alpha rhythm dominating the awake resting-state, and suggests distinct generative mechanisms for the cascading process during NREM sleep, a physiological state dominated by delta waves.

The coexistence of neuronal avalanches and oscillations was investigated in mature cortex slice cultures and in rodents, where nested $\theta / \beta / \gamma$-oscillations embedded in avalanches were reported [22, 32 ] and $\theta$ oscillations were linked to large avalanches [32]. In line with our observations pointing to substantial differences between cascading processes during distinct physiological states - i.e. awake resting-state and NREM sleep-, studies of spontaneous activity in mature cortex slice cultures showed that avalanche dynamics are highly sensitive to the excitation-inhibition balance [33, 30]. Furthermore, a temporal structure of neuronal avalanches consistent with the Omori law was also identified [47]. Recently, the relation between neuronal avalanches, $\gamma$ oscillations, and emergent signatures of critical dynamics has been studied in non-human primates [39]. On the other hand, in the human brain, the relationship between avalanche dynamics and oscillations had not been scrutinized to date. Indeed, simultaneous investigations of oscillations and avalanches in the human brain selectively focused on long-range temporal correlations in alpha amplitude fluctuations and on avalanche scaling features [43, 71]. At the same time, models capturing the simultaneous emergence of avalanches and oscillations concentrate on generic underlying mechanisms and signatures of criticality [48, 17, 24, 34]. In particular, a quantitative analysis of awake resting-state brain activity through a novel class of adaptive neural networks recently linked this coexistence to proximity to a non-equilibrium critical point at the onset of self-sustained oscillations [14, 34. In this context, the present study establishes the first functional and dynamical links between neural oscillations and avalanches in the awake resting-state, uncovering a deep relationship between two collective phenomena with antithetic features - scale-free avalanches and scale-specific brain rhythms. 
Overall, our analysis of accessible, near-synchronous collective behaviors provides a comprehensive view of resting-state dynamics underlying the dominant alpha rhythm in terms of a unique principle - the attenuation-amplification principle. This principle connects two previously unrelated characteristics attributed to alpha waves - pulsed inhibition and "waxing and waning" —, showing that they are part of a single dynamic organization based on attenuation-amplification mechanisms, a distinctive feature of the awake resting-state. Future works will focus on the role of AAD in information processing. In this respect, the approach we put forward will allow to (i) directly verify, and potentially extend, the pulsed inhibition hypothesis [37] through the analysis of the AAD in relation to processing of sensory stimuli, and (ii) clarify the functional role of the "waxing and waning" phenomena. More generally, our approach outlines a coherent view for the dichotomy of scale-specific oscillations and scale-free avalanches [22, 32, 30, 39], and demonstrates a functional and informative connection between these two phenomena. This may prove useful to dissect collective neural dynamics underlying brain oscillations in all those contexts where simultaneous recordings of single-cells and coarse-grained signals are out of reach, harvesting information from the analysis of neural cascading processes that would not be accessible otherwise.

\section{Materials and Methods}

\section{Data acquisition and pre-processing}

MEG of the resting state. This study uses data sets previously collected at the NIMH. The 4-min MEG data are the same as used in [60]. The 40-min data are the same as used in [69]. Ongoing brain activity was recorded from 100 healthy participants in the MEG core facility at the NIMH (Bethesda, MD, USA) for a duration of 4 min (eyes closed), and from three healthy female participants for a duration of $40 \mathrm{~min}$ (eyes closed). All experiments were carried out in accordance with NIH guidelines for human subjects. The sampling rate was $600 \mathrm{~Hz}$, and the data were band-pass filtered between 1 and $80 \mathrm{~Hz}$. Power-line interferences were removed using a 60-Hz notch filter designed in Matlab (Mathworks). The sensor array consisted of 275 axial first-order gradiometers. Two dysfunctional sensors were removed, leaving 273 sensors in the analysis. Analysis was performed directly on the axial gradiometer waveforms. For the present studies 73 subjects $[70(4 \mathrm{~min})+3(40 \mathrm{~min})]$ with a dominant alpha peak and AAD transition were selected.

EEG of the resting state Resting-state EEG was recorded for 3 min from six right-handed healthy volunteers. Participants had no history of neurological or psychiatric diseases and had normal or corrected-to-normal vision. All gave written informed consent, and were paid for their participation. The study was approved by a local ethics committee (Ben-Gurion University) and was in accordance with the ethical standards of the Declaration of Helsinki. EEG was recorded using the g.tec HIamp system (g.tec, Austria) with 64 gel-based electrodes ( $\mathrm{AgCl}$ electrolyte gel). Electrodes were positioned according to the standard 10/20 system with linked ears reference. Impedances of all electrodes were kept below $5 \mathrm{k} \Omega$. Data were pre-processed using a combination of the EEGLAB Matlab toolbox [16] routines and custom code. After high-pass filtering (cut-off $1 \mathrm{~Hz}$ ), a customized adaptive filter was applied to suppress line-noise. This was followed by Artifact Subspace Reconstruction [42], rereferencing to the mean, and low-pass filtering (cutoff $60 \mathrm{~Hz}$ ). Subsequently, an ICA (independent component analysis) algorithm was applied to the data [6]. The resulting ICs were evaluated automatically for artifacts by combining spatial, spectral and temporal analysis of ICs. ICs identified as 
containing ocular, muscular, or cardiac artifacts were removed from the data.

Sleep EEG The data analyzed in this study were extracted from overnight polysomnography (PSG) recordings acquired at the Parma Sleep Disorders Centre. Ten healthy subjects, 5 males and 5 females, mean aged 39,6 years (age range 28-53), were selected after an entrance investigation based on the following inclusion criteria: (i) absence of any psychiatric, medical, and neurological disorders; (ii) normal sleep/wake habits without any difficulties in falling or remaining asleep at night. A personal interview integrated by a structured questionnaire confirmed good vigilance level during the daytime; and (III) no drug intake at the time of the PSG or the month before. Full-night unattended PSG recordings were performed with EOG (2 channels), EEG (19 channels in 7 subjects, $\mathrm{Ag} / \mathrm{AgCl}$ electrodes placed according to the 10-20 International System referred to linked-ear lobes: Fp2, F4, C4, P4, O2, F8, T4, T6, Fz, Cz, Pz, Fp1, F3, C3, P3, O1, F7, T3, T5; 25 channels in 3 subjects: CP3, CP4, C5, C6, C2, C1, FC4, FC3, F4, C4, P4, O2, F8, T4, T6, Fz, Cz, Pz, F3, C3, P3, O1, F7, T3, T5), EMG of the submentalis muscle, ECG, body position monitor, and signal for SpO2 (pulse-oximetry O2-saturation). Sleep EEG recordings were obtained using a Brain Quick Micromed System 98 (Micromed, SPA) recording machine. The institutional Ethical Committee Area Vasta Emilia Nord approved the study (protocol no. 19750).

\section{Analysis of collective neural dynamics}

Resting wakefulness For each MEG (EEG) sensor, positive and negative deflections in the MEG (EEG) signal were detected by applying a threshold $h$ at $\pm n \mathrm{SD}$. Comparison of the signal distribution to the best fit Gaussian indicates that the two distributions start to deviate from one another around 2.7SD [60]. Thus, thresholds smaller than 2.7SD will lead to the detection of many events related to noise in addition to real events, whereas much larger thresholds will miss many of the real events. To avoid noise-related events while preserving most of relevant events, in this study, we used threshold values $h>2.9 \mathrm{SD}$. To ensure a similar event rate across different sets of recordings (4-min MEG, 40-min MEG, 3-min EEG), we used the following $h$ values: 3.3 SD for 4-min MEG; 3 SD for 40-min MEG, and 3 SD for 3-min EEG. In each excursion beyond the threshold, a single event was identified at the most extreme value (maximum for positive excursions and minimum for negative excursions). Data were binned using a time window $\epsilon=2 T_{\text {meg }}=3.3 \mathrm{~ms}$ and $\epsilon=T_{e e g}=4 \mathrm{~ms}$ for MEG and EEG data, respectively. $T_{m e g}=1.67 \mathrm{~ms}$ was the sampling interval for MEG recordings, while $T_{e e g}=4 \mathrm{~ms}$ was the sampling interval for EEG recordings. An avalanche was defined as a continuous sequence of time bins in which there was at least one event on any sensor, ending with a time bin with no events on any sensor. The size of an avalanche, $s$, was defined as the number of events in the avalanche. For more details, see [60]. The size of an avalanche can be equivalently defined as the sum over all channels of the absolute values of the signals exceeding the threshold (Fig. S1).

Sleep Sleep was scored visually in 30-s epochs using standard criteria [50]. To identify avalanches during sleep, positive and negative deflections were detected by applying a threshold $h \pm 2 \mathrm{SD}$ (comparison of the signal distribution to the best fit Gaussian indicates that the two distributions start to deviate from one another at around 2SD). An avalanche was then defined as a continuous time interval in which there was at least one EEG channel supra-threshold. Due to the reduced number of electrodes in EEG sleep recordings, the size of an avalanche was defined as the sum over all channels of the absolute values of the signals exceeding the threshold. This definition is equivalent to the 
definition of avalanche size as the number of electrodes with positive (negative) deflections exceeding the threshold $h$ [4] (Fig. S1).

Conditional probability Each recording results in a sequence of avalanches $a_{i}$, and corresponding sizes $s_{i}$ (Fig. 1). The quantity $\Delta s=s_{i+1}-s_{i}$ is the difference between the sizes of two consecutive avalanches $a_{i}$ and $a_{i+1}$, and is used to study their monotonic relation-i.e. whether $s_{i}$ is more likely to be smaller or larger than $s_{i+1}$-as a function of the quiet times $\Delta t$ occurring in between. To this end, the following conditional probability is defined,

$$
P\left(\Delta s<s_{0} \mid \Delta t<t_{0}\right) \equiv \frac{N\left(\Delta s<s_{0}, \Delta t<t_{0}\right)}{N\left(\Delta t<t_{0}\right)}
$$

where $N\left(\Delta s<s_{0}, \Delta t<t_{0}\right)$ is the number of avalanche couples which are separated by a quiet time $\Delta t$ shorter than a given $t_{0}$ and whose size difference $\Delta s$ is smaller than a given $s_{0}$, and $N\left(\Delta t<t_{0}\right)$ is the number of quiet times $\Delta t$ shorter than $t_{0} . P\left(\Delta s<s_{0} \mid \Delta t<t_{0}\right)$ gives the probability that two consecutive avalanches separated by a $\Delta t$ shorter than $t_{0}$ are such that $\Delta s<s_{0}$, with $s_{0}$ a positive or negative integer. The conditional probability defined by Eq.(1) assesses the monotonic relation between consecutive avalanches as a function of the quiet time separating them, thus, providing a detailed picture of the correlation landscape of the avalanche process. To measure the strength and significance of such a relationship, for each given couple of thresholds $s_{0}$ and $t_{0}, P\left(\Delta s<s_{0} \mid \Delta t<t_{0}\right)$ is systematically compared with the same conditional probability evaluated over $10^{4}$ independent surrogate avalanche time series. Surrogates are obtained by randomly reshuffling avalanche sizes while keeping fixed their starting and ending times. $P\left(\Delta s<s_{0} \mid \Delta t<t_{0}\right)$ are then compared with the average surrogate conditional probabilities, $P^{*}\left(\Delta s<s_{0} \mid \Delta t<t_{0}\right)$, by analyzing the quantity

$$
\delta P\left(s_{0}, t_{0}\right) \equiv P\left(\Delta s<s_{0} \mid \Delta t<t_{0}\right)-P^{*}\left(\Delta s<s_{0} \mid \Delta t<t_{0}\right)
$$

as a function of $s_{0}$ for different fixed values of $t_{0}$ (Fig. 3). Being $\sigma^{*}\left(s_{0}, t_{0}\right)$ the standard deviation of the mean surrogate conditional probability $P^{*}\left(\Delta s<s_{0} \mid \Delta t<t_{0}\right)$, if $\left|\delta P\left(s_{0}, t_{0}\right)\right|>2 \sigma^{*}\left(s_{0}\right.$, $\left.t_{0}\right)$, one can conclude that $P$ and $P^{*}$ are significantly different (0.05 significance level), and that significant correlations exist between $\Delta s<s_{0}$ and $\Delta t<t_{0}$.

Two cases must be distinguished: $\delta P\left(s_{0}, t_{0}\right)>0$ and $\delta P\left(s_{0}, t_{0}\right)<0$. If $\delta P\left(s_{0}, t_{0}\right)>0$, the number of avalanche couples $N\left(\Delta s<s_{0}, \Delta t<t_{0}\right)$ satisfying both conditions is significantly larger in the original data than in the surrogates; namely, it is more likely to find couples satisfying both conditions in the original rather than in the surrogate avalanche time series. Hence, $\Delta s$ and $\Delta t$ are positively correlated. On the contrary, if $\delta P\left(s_{0}, t_{0}\right)<0$, the number of couples $N\left(\Delta s<s_{0}, \Delta t<t_{0}\right)$ satisfying both conditions is significantly larger in the surrogates than in the original data; namely, it is more likely to find couples satisfying both conditions in the uncorrelated surrogates rather than in the real avalanche time series. In this case, one says that $\Delta s$ and $\Delta t$ are negatively correlated.

Omori law The number of avalanches per unit time, $N(t)$, occurring after a time $t$ has elapsed from the main avalanche $A^{*}$ is computed using a time window $\delta t$ that increases logarithmically. Denoting a set of window boundaries as $W=\left(w_{1}, w_{2}, \ldots, w_{k}\right)$ and fixing $w_{1}=10 \mathrm{~ms}$, the logarithmic windows fulfill the relation $w_{i+1}=w_{i} \cdot 10^{c}$, which implies that the window size is constant in logarithmic scale, i.e. $\log w_{i+1}-\log w_{i}=c$. The following window sizes of $c$ have been used in this study: $c=0.1$ for the $N(t)$ of the awake resting-state (Fig. 3, Fig. S4); $c=0.11$ for the $N(t)$ during sleep (Fig. 4). 
Spearman's correlation coefficient Given two variables $X$ and $Y$, the Spearman's correlation coefficient is defined as

$$
\rho_{s}=\frac{\operatorname{cov}\left(r g_{X}, r g_{Y}\right)}{\sigma_{r g_{X}} \sigma_{r g_{Y}}}
$$

where $r g_{X}$ and $r g_{Y}$ are the tied rankings of $X$ and $Y$, respectively, $\sigma_{r g_{X}}$ and $\sigma_{r g_{Y}}$ their standard deviations, and $\operatorname{cov}\left(r g_{X}, r g_{Y}\right)$ indicates the covariance between $r g_{X}$ and $r g_{Y}$.

Surrogate signals Surrogate signals are obtained by random phase shuffling of the original continuous signals. A Fourier transform of each sensor signal is performed, and the corresponding phases are randomized while amplitudes are preserved. The surrogate signals are then obtained by performing an inverse Fourier transform. The random phase shuffling destroys phase synchronization across cortical sites while preserving the linear properties of the original signals, such as power spectral density and two-point correlations 62 .

\section{Acknowledgements}

FL acknowledges support from the European Union's Horizon 2020 research and innovation program under the Marie Sklodowska-Curie Grant Agreement No. 754411. LdA acknowledges the Italian MIUR project PRIN2017WZFTZP for financial support and the project E-PASSION of the program VALERE 2019 funded by the University of Campania, Italy "L. Vanvitelli". OS acknowledges support from the Israel Science Foundation, Grant No. 504/17. Supported in part by DIRP ZIAMH02797 to DP. 
bioRxiv preprint doi: https://doi.org/10.1101/2022.03.03.482657; this version posted March 4, 2022. The copyright holder for this preprint (which was not certified by peer review) is the author/funder, who has granted bioRxiv a license to display the preprint in perpetuity. It is made available under aCC-BY-NC-ND 4.0 International license.

\section{Supplementary Information}

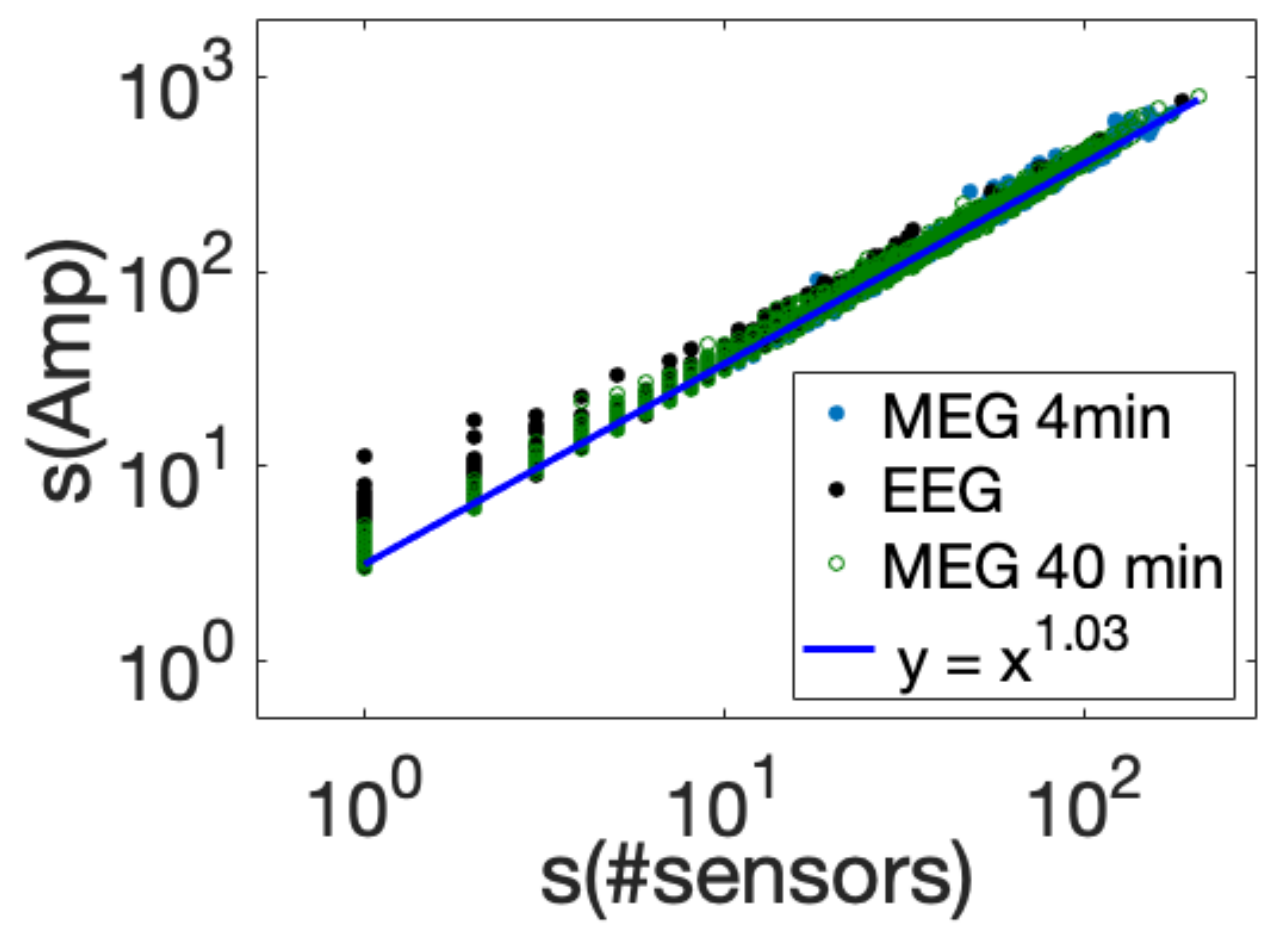

Fig. S1: Scatter plot between avalanche sizes $s$ (\#sensors) measured as number of active sensors, and avalanche sizes $s(A m p)$ measured as the sum of the signal amplitudes across active sensors in MEG and EEG recordings of an individual subject. We find that $s(A m p) \propto s(\# \text { sensor })^{1.03}$. This implies that the definition of avalanche size as the number of active sensors is equivalent to the definition based on the sum of the signal amplitudes across active sensors in both in MEG and EEG recordings (Materials and Methods). 

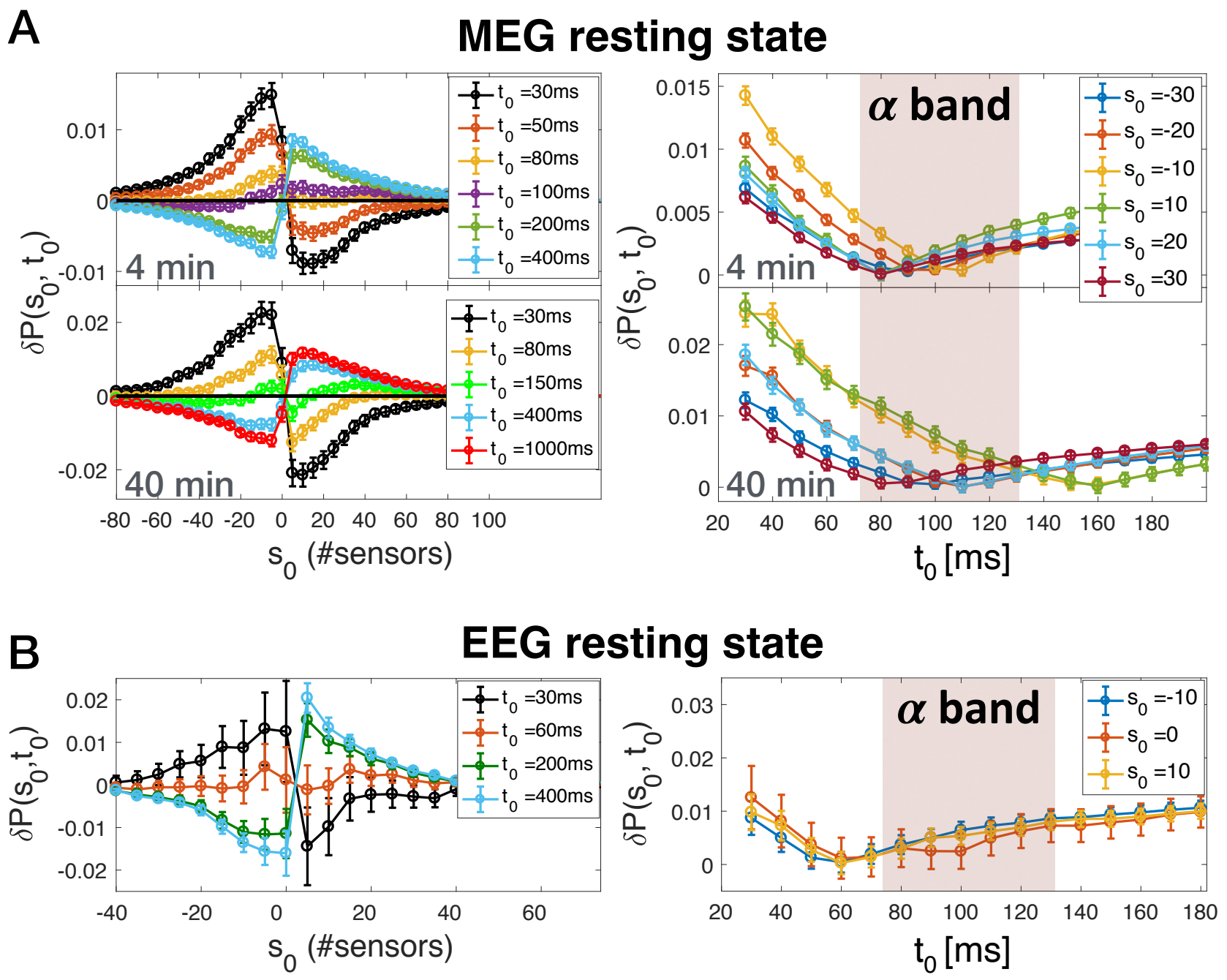

Fig. S2: The transition from the attenuation to the amplification regime in the resting-state cascading process occurs at the characteristic time of the alpha rhythm. (A) (Left panel) $\delta P\left(s_{0}, t_{0}\right)$ as a function of the threshold $s_{0}$ on $\Delta s$ for different values of the threshold $t_{0}$ on the quiet times $\Delta t$ 's for MEG resting state recordings. The error bar on each data point is two times the standard deviation $\sigma^{*}$ associated with the surrogates $P\left(s_{0}, t_{0}\right)^{*}$ (see Fig. 2 and Materials and Methods). For a given threshold $t_{0}$, the maximum in $\delta P\left(s_{0}, t_{0}\right)$ indicates the preferred relation between consecutive avalanches separated by quiet times shorter than $t_{0}$. We notice that, both in the 4-min (upper panel) and 40-min MEG recordings (lower panel), for $t_{0}<100 \mathrm{~ms}, \delta P\left(s_{0}, t_{0}\right)$ has a maximum at $s_{0}<0$, and therefore an avalanche tends to be smaller than its preceding one $\left(s_{i+1}<s_{i}\right.$, attenuation regime). On the other hand, for $t_{0}>100$ ms the maximum moves towards positive $s_{0}$, implying that a given avalanche tends to be larger than the preceding one $\left(s_{i+1}>s_{i}\right.$, amplification regime). Remarkably, for $t_{0} \simeq 100 \mathrm{~ms}, \delta P\left(s_{0}, t_{0}\right)$ is very close to zero for each $s_{0}$, with values that are not significantly different among each other. This indicates that the conditional probabilities evaluated on the original data are very close to those calculated from the surrogates. Hence, we find that at $t_{0} \simeq 100 \mathrm{~ms}$ there is not a preferred sign for $\Delta s$, indicating that $\Delta t=100 \mathrm{~ms}$ is a transition point from one dynamical regime to another. (Right panel) The absolute value $\left|\delta P\left(s_{0}, t_{0}\right)\right|$ as a function of $t_{0}$ for several fixed values of $s_{0}$. For each $s_{0},\left|\delta P\left(s_{0}, t_{0}\right)\right|$ has a minimum around $t_{0}=100 \mathrm{~ms}$. More specifically, the minimum is always comprised between $80 \mathrm{~ms}$ and $110 \mathrm{~ms}$ for the 4-min recordings (upper panel), and between $80 \mathrm{~ms}$ and $160 \mathrm{~ms}$ for the 40-min recordings (lower panel). Such time scales correspond to the characteristic period of the $\alpha$ rhythm. (B) (Left panel) $\delta P\left(s_{0}, t_{0}\right)$ as a function of the threshold $s_{0}$ on $\Delta s$ for different values of the threshold $t_{0}$ on the quiet times $\Delta t$ 's for EEG resting state recordings. As in MEG recordings, we observe a transition between two different dynamical regimes. For $t_{0}<60 \mathrm{~ms}, \delta P\left(s_{0}, t_{0}\right)$ has a maximum at $s_{0}<0$, and therefore an avalanche tends to be smaller than its preceding one $\left(s_{i+1}<s_{i}\right.$, attenuation regime). On the other hand, for $t_{0}>60 \mathrm{~ms}$ the maximum moves towards positive $s_{0}$, implying that a given avalanche tends to be larger than the preceding one $\left(s_{i+1}>s_{i}\right.$, amplification regime). Remarkably, for $t_{0} \simeq 60 \mathrm{~ms}, \delta P\left(s_{0}, t_{0}\right)$ is very close to zero for each $s_{0}$, with values that are not significantly different among each other. This indicates that the conditional probabilities evaluated on the original data are very close to those calculated from the surrogates. Hence, we find that at $t_{0} \simeq 60 \mathrm{~ms}$ there is not a preferred sign for $\Delta s$, suggesting indeed that $\Delta t=60 \mathrm{~ms}$ is a transition point from one dynamical regime to another. (Right panel) The absolute value $\left|\delta P\left(s_{0}, t_{0}\right)\right|$ as a function of $t_{0}$ for several fixed values of $s_{0}$. For each $s_{0},\left|\delta P\left(s_{0}, t_{0}\right)\right|$ has a minimum around $t_{0}=60 \mathrm{~ms}$. 


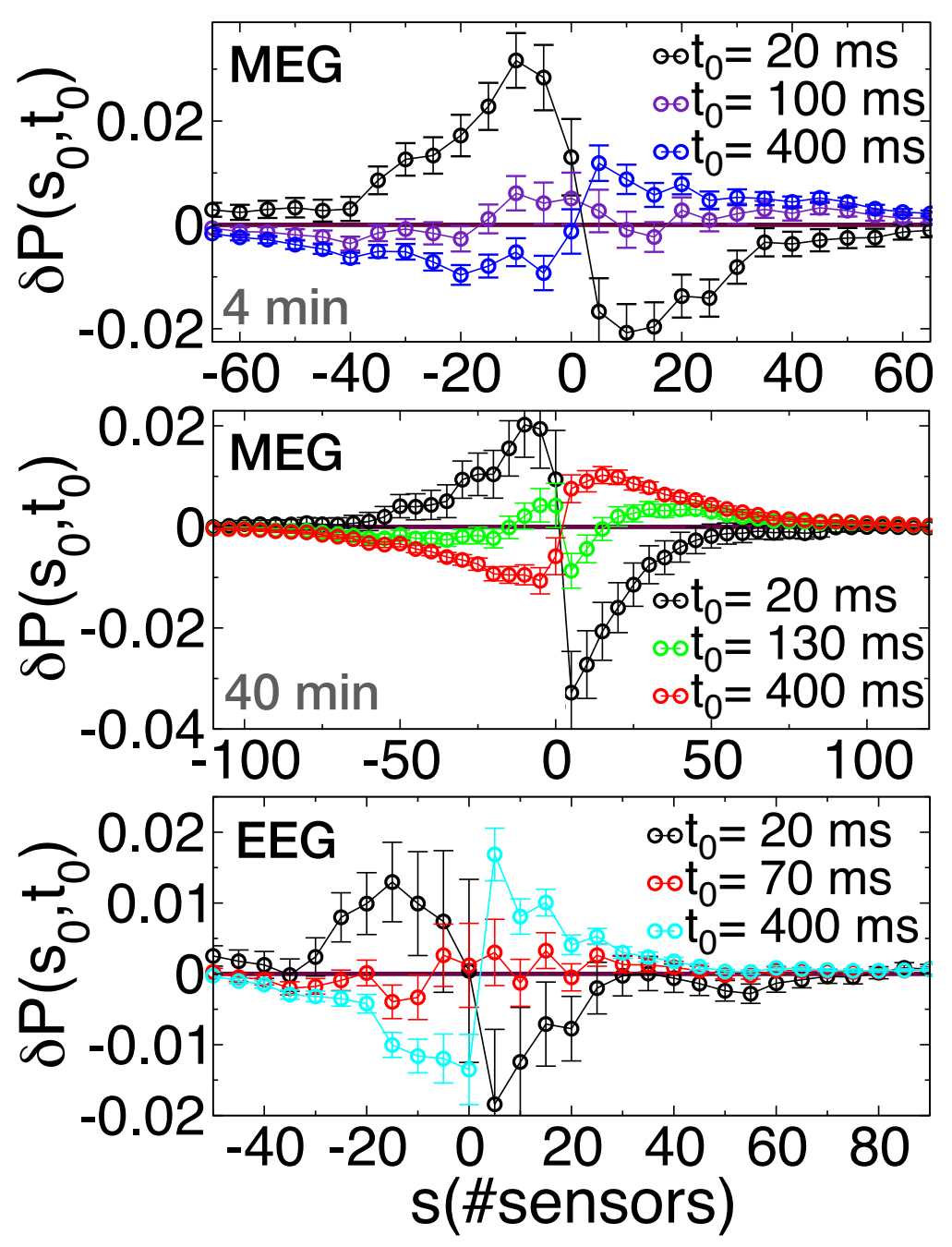

Fig. S3: The attenuation-amplification transition in the neural cascading dynamics is robust and consistent across individual subjects. (Top) $\delta P\left(s_{0}, t_{0}\right)$ as a function of the threshold $s_{0}$ for an individual 4-min MEG recording. (Middle) $\delta P\left(s_{0}, t_{0}\right)$ as a function of the threshold $s_{0}$ for an individual 40-min MEG recording. (Bottom) $\delta P\left(s_{0}, t_{0}\right)$ as a function of the threshold $s_{0}$ for an individual EEG recording. The error bar on each data point is two times the standard deviation $\sigma^{*}$ associated with the surrogates $P^{*}\left(s_{0}, t_{0}\right)$ (Materials and Methods). For a given threshold $t_{0}$, the maximum in $\delta P\left(s_{0}, t_{0}\right)$ indicates the preferred relation between consecutive avalanches separated by quiet times shorter than $t_{0}$. In all the three cases, we notice that for $t_{0}<100 \mathrm{~ms} \delta P\left(s_{0}, t_{0}\right)$ has a maximum at $s_{0}<0$, and therefore an avalanche tends to be smaller than its preceding one $\left(s_{i+1}<s_{i}\right.$, attenuation regime). On the other hand, for $t_{0}>100 \mathrm{~ms}$ the maximum moves towards positive $s_{0}$, implying that a given avalanche tends to be larger than the preceding one $\left(s_{i+1}>s_{i}\right.$, amplification regime). Remarkably, for $t_{0} \simeq 100 \mathrm{~ms}, \delta P\left(s_{0}, t_{0}\right)$ is very close to zero for each $s_{0}$, indicating that the conditional probabilities evaluated on the original data are very close to those calculated from the surrogates. Hence, we find that at $t_{0} \simeq 100 \mathrm{~ms}$ there is not a preferred sign for $\Delta s$. This indicates that, both in pooled data and individual subjects, $\Delta t \approx 100 \mathrm{~ms}$ is a transition point from the attenuation to the amplification regime. 

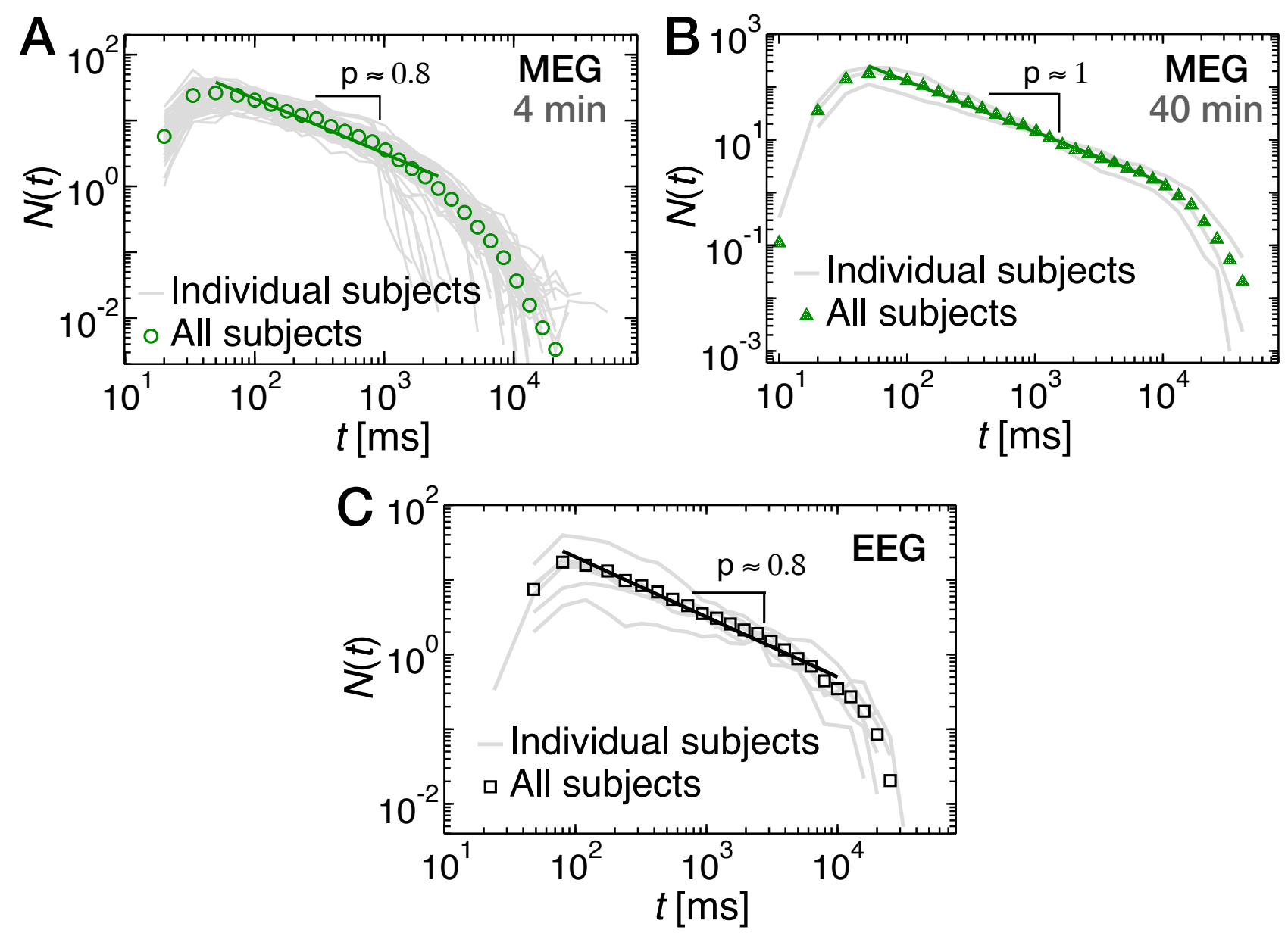

Fig. S4: The number of avalanches per unit time, $N(t)$, occurring after a main avalanche $\left(A^{*}>30\right)$ obeys the Omori law. $N(t)$ decreases roughly as the reciprocal of the time $t$ elapsed from the main shock, i.e. $N(t) \propto t^{-p}$. (A) $N(t)$ for 4-min MEG recordings from individual subjects (grey lines), and pooled subjects (green circles). Powerlaw fit (green thick line): $p=0.8326 \pm 0.0453$. (B) $N(t)$ for 40-min MEG recordings from individual subjects (grey lines), and pooled subjects (green triangles). Power-law fit (green thick line): $p=0.9596 \pm 0.0137$. (C) $N(t)$ for EEG recordings from individual subjects (grey lines), and pooled subjects (black squares). Power-law fit (black thick line): $p=0.8041 \pm 0.0277$. 
bioRxiv preprint doi: https://doi.org/10.1101/2022.03 03.482657; this version posted March 4 2022. The copyright holder for this preprint (which was not certified by peer review) is the author/funder, who has granted bioRxiv a license to display the preprint in perpetuity. It is made available under aCC-BY-NC-ND 4.0 International license.

\section{References}

[1] P. Allegrini, D. Menicucci, R. Bedini, L. Fronzoni, A. Gemignani, P. Grigolini, B. J. West, and P. Paradisi. Spontaneous brain activity as a source of ideal 1/f noise. Phys. Rev. E, 80:061914, 2009.

[2] R. P. Bartsch, K. K. Liu, A. Bashan, and P. Ch. Ivanov. Network physiology: How organ systems dynamically interact. PLoS One, 10:e0142143, 2015.

[3] A. Bashan, R. P. Bartsch, J. W. Kantelhardt, S. Havlin, and P. Ch. Ivanov. Network physiology reveals relations between network topology and physiologic function. Nat. Comm., 3:702, 2012.

[4] J. M. Beggs and D. Plenz. Neuronal avalanches in neocortical circuits. J. Neurosci., 23:1116711177, 2003.

[5] C. G. D. Behn, E. N. Brown, T. E. Scammell, and N. J. Kopell. Mathematical model of network dynamics governing mouse sleep-wake behavior. J. Neurophysiol., 97(6):3828-3840, Jun 2007.

[6] A. J. Bell and T. J. Sejnowski. An information-maximization approach to blind separation and blind deconvolution. Neural Comput., 7:1129-1159, 1995.

[7] H. Berger. Über das elektrenkephalogramm des menschen. Arch.Psychiatr.Nervenkr., 87:527-570, 1929.

[8] G. Buzsaki and A. Draguhn. Neuronal oscillations in cortical networks. Science, 304:1926-1929, 2004.

[9] G. Buzsaki and B. O. Watson. Brain rhythms and neural syntax: implications for efficient coding of cognitive content and neuropsychiatric disease. Dialogues Clin. Neurosci., 14(4):345-367, 2012.

[10] A. V. Chechkin, R. Metzler, J. Klafter, and V. Y. Gonchar. Introduction to the theory of Levy flights. Anomalous Transport: Foundations and Applications. Wiley-VCH Verlag GmbH \& Co. KGaA, 2008.

[11] M. S. Clayton, N. Yeung, and R. C. Kadosh. The many characters of visual alpha oscillations. Euro. J. Neurosci., 48:2498-2508, 2018.

[12] L. de Arcangelis, C. Godano, J. R. Grasso, and E. Lippiello. Statistical physics approach to earthquake occurrence and forecasting. Phys. Rep., 628:1-92, 2016.

[13] L. de Arcangelis, C. Godano, E. Lippiello, and M. Nicodemi. Universality in solar flare and earthquake occurrence. Phys. Rev. Lett., 96:051102, 2006.

[14] D. De Martino. Feedback-induced self-oscillations in large interacting systems subjected to phase transitions. J. Phys. A, 52:045002, 2019.

[15] G. Deco, M. L. Kringelbach, V. K. Jirsa, and P. Ritter. The dynamics of resting fluctuations in the brain: metastability and its dynamical cortical core. Sci. Rep., 7:3095, 2017.

[16] A. Delorme and S. Makeig. Eeglab: an open source toolbox for analysis of single-trial eeg dynamics including independent component analysis. J. Neurosci. Meth., 134(1):9-21, 2004. 
bioRxiv preprint doi: https://doi.org/10.1101/2022 03.03 .482657 t this version posted March 4 2022. The copyright holder for this preprint (which was not certified by peer review) is the author/funder, who has granted bioRxiv a license to display the preprint in perpetuity. It is made available under aCC-BY-NC-ND 4.0 International license.

[17] S. Di Santo, P. Villegas, R. Burioni, and M. A. Munoz. Landau-Ginzburg theory of cortex dynamics: Scale-free avalanches emerge at the edge of synchronization. Proc. Natl. Acad. Sci. USA, 115:E1356-E1365, 2018.

[18] A. J. Fontenele, N. A. P. de Vasconcelos, T. Feliciano, L. A. A. Aguiar, C. Soares-Cunha, B. Coimbra, L. D. Porta, S. Ribeiro, A. J. Rodrigues, N. Sousa, P. V. Carelli, and M. Copelli. Criticality between cortical states. Phys. Rev. Lett., 122(20):208101, 2019.

[19] F. Freyer, K. Aquino, P. A. Robinson, P. Ritter, and M. Breakspear. Bistability and non-gaussian fluctuations in spontaneous cortical activity. J. Neurosci., 29(26):8512-8524, 2009.

[20] F. Freyer, J. A. Roberts, R. Becker, P. A. Robinson, P. Ritter, and M. Breakspear. Biophysical mechanisms of multistability in resting-state cortical rhythms. J. Neurosci., 31(17):6353-6361, 2011.

[21] P. Fries. A mechanism for cognitive dynamics: neuronal communication through neuronal coherence. Trends Cogn. Sci., 9(10):474-480, 2005.

[22] D. E. Gireesh and D. Plenz. Neuronal avalanches organized as nested theta-and beta/gammaoscillations during development of cortical layer 2/3. Proc Natl Acad Sci USA, 105(21):7576-7581, 2008.

[23] P. Ch. Ivanov, A. Yuen, B. Podobnik, and Y. Lee. Common scaling patterns in intertrade times of us stocks. Phys. Rev. E, 69(5):056107, 2004.

[24] O. Kinouchi, L. Brochini, A. A. Costa, J. G. F. Campos, and M. Copelli. Stochastic oscillations and dragon king avalanches in self-organized quasi-critical systems. Sci. Rep., 9:3874, 2019.

[25] W. Klimesch, P. Sauseng, and S. Hanslmayr. Eeg alpha oscillations: The inhibition-timing hypothesis. Brain Res. Rev., 53(1):63-88, 2007.

[26] P. Lakatos, A. S. Shah, K. H. Knuth, I. Ulbert, G. Karmos, and C. E. Schroeder. An oscillatory hierarchy controlling neuronal excitability and stimulus processing in the auditory cortex. $J$. Neurophysiol., 94:1904-1911, 2005.

[27] K. Linkenkaer-Hansen, V. V. Nikouline, J. M. Palva, and R. J. IImoniemi. Long-range temporal correlations and scaling behavior in human brain oscillations. J. Neurosci., 21(4):1370-1377, 2001.

[28] F. Lombardi and L. de Arcangelis. Temporal organization of ongoing brain activity. Euro. Phys. J. Special Topics, 223(11):2119-2130, 2014.

[29] F. Lombardi, M. Gomez-Extremera, P. Bernaola-Galvan, R. Vetrivelan, C. B. Saper, T. E. Scammell, and P. C. Ivanov. Critical dynamics and coupling in bursts of cortical rhythms indicate non-homeostatic mechanism for sleep-stage transitions and dual role of vlpo neurons in both sleep and wake. J. Neurosci., 40(1):171-190, 2020.

[30] F. Lombardi, H. J. Herrmann, and L. de Arcangelis. The Functional Role of Critical Dynamics in Neural Systems, chapter Avalanche Dynamics and Correlations in Neural Systems, pages 1-26. Springer, Cham, 2019 . 
[31] F. Lombardi, H. J. Herrmann, C. Perrone-Capano, D. Plenz, and L. de Arcangelis. Balance between excitation and inhibition controls the temporal organization of neuronal avalanches. Phys. Rev. Lett, 108:228703, 2012.

[32] F. Lombardi, H. J. Herrmann, D. Plenz, and L. de Arcangelis. On the temporal organization of neuronal avalanches. Front. Syst. Neurosci., 8:204. doi:10.3389/fnsys.2014.00204, 2014.

[33] F. Lombardi, H. J. Herrmann, D. Plenz, and L. de Arcangelis. Temporal correlations in avalanche occurrence. Sci. Rep., 6:24690, 2016.

[34] F. Lombardi, S. Pepić, O. Shriki, G. Tkačik, and D. De Martino. Quantifying the coexistence of neuronal oscillations and avalanches. arXiv:2108.06686, 2021.

[35] F. Lombardi, O. Shriki, H. J. Herrmann, and L. de Arcangelis. Long-range temporal correlations in the broadband resting state activity of the human brain revealed by neuronal avalanches. Neurocomputing, 461:657-666, 2021.

[36] F. Lombardi, J. W. Wang, X. Zhang, and P. Ch. Ivanov. Power-law correlations and coupling of active and quiet states underlie a class of complex systems with self-organization at criticality. EPJ web of conferences, 230:00005, 2020.

[37] K. E. Mathewson, A. Lleras, D. M. Beck, M. Fabiani, T. Ro, and G. Gratton. Pulsed out of awareness: Eeg alpha oscillations represent a pulsed-inhibition of ongoing cortical processing. Front. Psychol., 2:99, 2011.

[38] A. Mazzoni, F. D. Broccard, E. Garcia-Perez, P. Bonifazi, E. M.Ruaro, and V. Torre. On the dynamics of the spontaneous activity in neuronal networks. PLoS ONE, 2(5):e439, 2007.

[39] S. R. Miller, S. Yu, and D. Plenz. The scale-invariant, temporal profile of neuronal avalanches in relation to cortical $\gamma$-oscillations. Sci. Rep., 9:16403, 2019.

[40] K. Mimura. On the periodic fluctuations of alpha waves. Jap. J. Physiol., 21:375-386, 1971.

[41] K. Motokawa. Die analyse der perioden im normalen elektrenkephalogramm des menschen. Tohoku J. exp. Med., 42:9-21, 1942.

[42] T. R. Mullen, C. A. Kothe, Y. M. Chi, A. Ojeda, T. Kerth, S. Makeig, and C. G. Jung, T P. Real-time neuroimaging and cognitive monitoring using wearable dry eeg. IEEE Trans. Biomed. Eng., 62(11):2553-67, 2015.

[43] J. M. Palva, A. Zhigalov, J. Hirvonena, O. Korhonena, K. Linkenkaer-Hansen, and S. Palva. Neuronal long-range temporal correlations and avalanche dynamics are correlated with behavioral scaling laws. Proc. Natl. Acad. Sci. USA, 110(9):3585, 2013.

[44] V. Pasquale, P. Massobrio, L. L. Bologna, M. Chiappalone, and S. Martinoia. Self-organization and neuronal avalanches in networks of dissociated cortical neurons. J.Neurosci., 153:1354-1369, 2008.

[45] T. Petermann, T. C. Thiagarajan, M. Lebedev, M. Nicolelis, D. R. Chialvo, and D. Plenz. Spontaneous cortical activity in awake monkeys composed of neuronal avalanches. Proc. Natl. Acad. Sci. USA, 106(37):15921-15926, 2009. 
[46] G. Pfurtscheller, A. J. Stancak, and C. Neuper. Event-related synchronization (ers) in the alpha band - an electrophysiological correlate of cortical idling: a review. Int. J. Psychophysiol., 122(12):39-46, 1996.

[47] D. Plenz. Neuronal avalanches and coherence potentials. Eur. Phys. J. Special Topics, 205:259$301,2012$.

[48] S.-S. Poil, R. Hardstone, H. D. Mansvelder, and K. Linkenkaer-Hansen. Critical-state dynamics of avalanches and oscillations jointly emerge from balanced excitation/inhibition in neuronal networks. J. Neurosci., 32(29):9817-9823, 2012.

[49] A. Ponce-Alvarez, A. Jouary, M. Privat, G. Deco, and G. Sumbre. Whole-brain neuronal activity displays crackling noise dynamics. Neuron, 100:1446-1459, 2018.

[50] K. A. Rechtschaffen A. A manual of standardized terminology, techniques and scoring system for sleep stages of human subjects. U.S. Dept. of Health, Education, and Welfare, Bethesda, MD, 1968.

[51] T. L. Ribeiro, M. Copelli, F. Caixeta, H. Belchior, D. R. Chialvo, M. A. Nicolelis, and S. Ribeiro. Spike avalanches exhibit universal dynamics across the sleep-wake cycle. PLoS One, 5(11):e14129, 2010.

[52] A. K. Roopun, M. A. Kramer, L. M. Carracedo, M. Kaiser, C. H. Davies, R. D. Traub, N. J. Kopell, and M. A. Whittington. Temporal interactions between cortical rhythms. Front. Neurosci., 2:34, 2008.

[53] D. Rybski, S. V. Buldyrev, S. Havlin, F. Liljeros, and H. A. Makse. Communication activity in a social network: relation between long-term correlations and inter-event clustering. Sci. Rep., $2: 560,2012$.

[54] Y. B. Saalmann, M. A. Pinsk, L. Wang, X. Li, and S. Kastner. The pulvinar regulates information transmission between cortical areas based on attention demands. Science, 337:753-756, 2012.

[55] S. Sadaghiani and A. Kleinschmidt. Brain networks and a-oscillations: structural and functional foundations of cognitive control. Trends Cogn. Sci., 20:805-817, 2016.

[56] J. Samaha, T. C. Sprague, and B. R. Postle. Decoding and reconstructing the focus of spatial attention from the topography of alpha-band oscillations. J. Cogn. Neurosci., 28:1090-1097, 2016 .

[57] C. B. Saper, P. M. Fuller, N. Pedersen, J. Lu, and T. E. Scammell. Sleep state switching. Neuron, 68:1023-1042, 2010.

[58] S. Scarpetta and A. De Candia. Alternation of up and down states at a dynamical phase-transition of a neural network with spatiotemporal attractors. Front. Syst. Neurosci., 8:88, 2014.

[59] S. Scarpetta, F. Giacco, F. Lombardi, and A. De Candia. Effects of poisson noise in a if model with stdp and spontaneous replay of periodic spatiotemporal patterns, in absence of cue stimulation. Biosystems, 112(3):258-264, 2013. 
bioRxiv preprint doi: https://doi.org/10.1101/2022 03.03 482657: this version posted March 4 2022. The copyright holder for this preprint (which was not certified by peer review) is the author/funder, who has granted bioRxiv a license to display the preprint in perpetuity. It is made available under aCC-BY-NC-ND 4.0 International license.

[60] O. Shriki, J. Alstott, F. Carver, T. Holroyd, R. N. A. Hanson, M. L. Smith, R. Coppola, E. Bullmore, and D. Plenz. Neuronal avalanches in the resting meg of the human brain. J. Neurosci., 33(16):7079-7090, 2013.

[61] E. Tagliazucchi, P. Balenzuela, D. Fraiman, and D. R. Chialvo. Criticality in large-scale brain fmri dynamics unveiled by a novel point process analysis. Front. Physiol., 3:15, 2012.

[62] J. Theiler, S. Eubank, A. Longtin, B. Galdrikian, and J. D. Farmer. Testing nonlinearity in time series: the method of surrogate data. Physica D, 58:77-94, 1992.

[63] T. Utsu. International Handbook of Earthquake and Engineering Seismology, volume 81A. Academic Press, 2002.

[64] S. Vijayan, S. Ching, P. L. Purdon, E. N. Brown, and N. J. Kopell. Thalamocortical mechanisms for the anteriorization of alpha rhythms during propofol-induced unconsciousness. J. Neurosci., 33(27):11070-11075, 2013.

[65] S. Vijayan and N. J. Kopell. Thalamic model of awake alpha oscillations and implications for stimulus processing. Proc. Natl. Acad. Sci. USA, 109(45):18553-18558, 2012.

[66] W. G. Walter. Intrinsic rhythms of the brain, chapter Section 1, Neurophysiology, pages 279-298. Amer. Physiol. Soc., Washington, D.C., 1960.

[67] J. W. J. L. Wang, F. Lombardi, X. Zhang, C. Anaclet, and P. Ch. Ivanov. Non-equilibrium critical dynamics of bursts in $\theta$ and $\delta$ rhythms as fundamental characteristic of sleep and wake micro-architecture. PLoS Comp. Biol., 15(11):e1007268, 2019.

[68] X.-J. Wang. Neurophysiological and computational principles of cortical rhythms in cognition. Phys. Rev., 90:1195-1268, 2010.

[69] S. Yu, H. Yang, O. Shriki, and D. Plenz. Universal organization of resting brain activity at the thermodynamic critical point. Front. Syst. Neurosci., 7:42, 2013.

[70] M. Zhengyu, G. G. Turrigiano, R. Wessel, and K. B. Hengen. Cortical circuit dynamics are homeostatically tuned to criticality in vivo. Neuron, 104:655-664, 2019.

[71] A. Zhigalov, G. Arnulfo, L. Nobili, S. Palva, and J. Matias Palva. Relationship of fast- and slow-timescale neuronal dynamics in human meg and seeg. J. Neurosci., 35(13):5385-5396, 2015. 